\title{
Flavor violating leptonic decays of $\tau$ and $\mu$ leptons in the Standard Model with massive neutrinos
}

\author{
G. Hernández-Tomé ${ }^{1,2, a}$, G. López Castro ${ }^{1, b}$, P. Roig ${ }^{1, c}$ \\ ${ }^{1}$ Departamento de Física, Centro de Investigación y de Estudios Avanzados del Instituto Politécnico Nacional, Apdo. Postal 14-740, 07000 México, \\ D.F., Mexico \\ ${ }^{2}$ CAFPE and Departamento de Física Teórica y del Cosmos, Universidad de Granada, E-18071 Granada, Spain
}

Received: 23 October 2018 / Accepted: 27 December 2018 / Published online: 29 January 2019

(C) The Author(s) 2019

\begin{abstract}
We have revisited the computations of the flavor violating leptonic decays of the $\tau$ and $\mu$ leptons into three lighter charged leptons in the Standard Model with massive neutrinos. We were driven by a claimed unnaturally large branching ratio predicted for the $\tau^{-} \rightarrow \mu^{-} \ell^{+} \ell^{-}(\ell=\mu, e)$ decays (Pham, Eur Phys J C 8:513 1999), which was at odds with the corresponding predictions for the $\mu^{-} \rightarrow e^{-} e^{-} e^{+}$ processes (Petcov, Sov J Nucl Phys 25:340 1977). In contrast with the prediction in [17], our results are strongly suppressed and in good agreement with the approximation made in Ref. [15], where masses and momenta of the external particles were neglected in order to deal with the loop integrals. However -as a result of keeping external momenta and masses in the computation of the dominant penguin and box diagrams- we even find slightly smaller branching fractions. Therefore, we confirm that any future observation of such processes would be an unambiguous manifestation of new physics beyond the Standard Model.
\end{abstract}

\section{Introduction}

Lepton flavor violating (LFV) processes are forbidden in the standard model (SM) [1-3] with massless neutrinos. However, the experimental evidence of neutrino oscillations [46] claims for an extended model with neutrino mass terms. For massive neutrinos, the mass matrix will be nondiagonal in the interaction (weak) basis, as occurs in the quark sector $[7,8]$, and the mixing of three light neutrinos could be described through the $3 \times 3$ unitary Pontecorvo-MakiNakagawa-Sakata (PMNS) matrix [9-11]. In such a scenario, charged LFV transitions could arise, for instance, from

\footnotetext{
a e-mail: ghernandez@ fis.cinvestav.mx;ghernandezt@correo.ugr.es

b e-mail: glopez@fis.cinvestav.mx

c e-mail: proig@fis.cinvestav.mx
}

one loop diagrams involving a couple of $W \ell v_{\ell}$ vertices with different flavor neutrinos each. However, it turns out natural having a strong suppression for this class of processes owing to a GIM-like mechanism [12], just as it has been reported for the $\mu^{-} \rightarrow e^{-} \gamma$ decay, with a prediction at an unobservably low rate: $B R\left(\mu^{-} \rightarrow e^{-} \gamma\right) \sim \mathscr{O}\left(10^{-55}\right)$ [13-15], which is far away from the capacity of any current or foreseen experimental facility.

By way of contrast, the prediction for the $\tau^{-} \rightarrow \mu^{-} \ell^{+} \ell^{-}$ $(\ell=\mu, e)$ decays given by Ref. [17] claims that the GIM cancellation for these processes is much milder and a value of $B R\left(\tau^{-} \rightarrow \mu^{-} \ell^{+} \ell^{-}\right) \geq 10^{-14}$ is reported. An updated evaluation using the amplitude derived in Ref. [17], employing the latest global fit results for neutrino mixing [18-21] yields a branching fraction $\sim 4 \times 10^{-16}$ for the three muon channel. Both values are still far away from the PDG upper bounds, $1.5 \times 10^{-8}$ (for $\ell=e$ ) and $2.1 \times 10^{-8}(\ell=\mu)$ at $90 \%$ confidence level. ${ }^{1}$ Similarly, we verified that using the values reported in Refs. [18-21] for the neutrino mixing parameters, Pham's result [17] would predict a $\mu^{-} \rightarrow e^{-} e^{+} e^{-}$branching ratio of $\sim 2 \times 10^{-21}$, larger than Petcov's prediction [15] ( $\sim 10^{-53}$ evaluated with updated neutrino masses and mixing parameters, see Table 1) by at least some thirty orders of magnitude. Again, the current upper limit on this decay channel $\left(1 \times 10^{-12}\right.$ at $90 \%$ C.L. [18]) is still far from testing Pham's result [17]. This author claims that this unexpectedly large estimation is due to the presence of a logarithmic divergent term depending on the neutrino mass, which comes from a one-loop diagram that involves two neutrino propagators (diag. (d) in our Fig. 1).

\footnotetext{
${ }_{1}^{1}$ More stringent bounds of $1.1 \times 10^{-8}$ and $1.2 \times 10^{-8}$, respectively, can be obtained by combining results of different experiments according to the HFLAV group [22]. Belle-II shall be able to set limits on the $\tau^{-} \rightarrow \mu^{-} \mu^{+} \mu^{-}$decay at the level of $3 \times 10^{-10}$ with their full data set $\left(50 \mathrm{ab}^{-1}\right)$ [24].
} 
Certainly, considering the effects or processes that arise from quantum corrections could involve divergent loop integrals. However, in any renormalizable theory, the possible divergences must vanish order by order (in the loop or effective field theory expansion) to be able to define (finite) observables. In fact, in a Quantum Field Theory the divergences can be classified into two types: ultraviolet (UV) and infrared divergences (IR). The former (UV) appear in the high-energy regime and they can be healed redefining the theory parameters, whereas the latter (IR) occur in the low-energy regime and can be classified in soft and collinear divergences, which cancel however in properly defined (IR-safe) observables $[25,26]$. We show that the seeming logarithmic divergent behavior of the LFV amplitude reported in Ref. [17] is not present, as the vanishing momentum transfer approximation considered in that paper lies outside the physical region. Consequently, the rates of $L^{-} \rightarrow \ell^{-} \ell^{\prime-} \ell^{\prime+}$ decays in the SM extended with massive neutrinos are extremely suppressed, in agreement with Ref. [15]. It is worth noting that, as neutrino oscillations, the LFV amplitudes must vanish in the limit of degenerate neutrinos. Moreover, according to the KLN theorem [25,26], the amplitude for massless neutrinos can go to zero, but it is impossible that it presents an IR divergence. This requirement is satisfied by the result of Ref. [15], but it is not the case in Ref. [17] which behaves as $\sum_{j} U_{L j} U_{\ell j}^{*} \log \left(m_{v} / m_{W}\right)$ for very small neutrino masses. Our result, as it will be shown below, satisfies the expected agreement with the previous consistency remarks.

In Sects. 2 and 3 we discuss in detail our computation of these processes and compare it to those in Refs. [15] and [17], showing explicitly why the approximation in the latter is unreliable, and reproducing the results of [15] when masses and momenta of the external particles are neglected at the amplitude level. In Sect. 4 we analyze the (quite good) numerical accuracy of this latter approximation. Finally, we state our conclusions in Sect. 5. Several appendices complete technical details of our calculation.

\section{Z-Penguin contribution emission from internal neutrino line}

The $L^{-} \rightarrow \ell^{-} \ell^{\prime-} \ell^{\prime+}$ decays can be induced through the diagrams depicted in Fig. 1. Since the main purpose of this work is to falsify the existence of the logarithmic divergent term claimed in Ref. [17], we first concentrate on the amplitude of the diagram (d). We have, however, verified the corresponding expressions for the loop integrals in Ref. [15] for the particular process $\mu^{-} \rightarrow e^{-} e^{-} e^{+}$, when masses and momenta of external leptons are neglected in the computations. Particularly, in Ref. [15] it is shown that the corresponding branching ratio is completely dominated by those diagrams with two neutrino propagators, i.e. (d) and (e) in Fig. 1, which contribute comparably.

In our analysis, we keep employing the convention used by Ref. [17], in order to denote the masses and momenta (see Fig. 1) of the external leptons, that is $M$ and $P$ ( $m$ and $p$ ) stand for the mass and momentum of the $L^{-}\left(\ell^{-}\right)$lepton, respectively. In this way, the amplitude of the diagram (d) can be written as

$\mathscr{M}_{d} \sim \frac{i}{m_{Z}^{2}} l_{L \ell}^{\lambda} \times l_{\ell^{\prime} \ell^{\prime} \lambda}$,

where $l_{\ell^{\prime} \ell^{\prime} \lambda}=-i g /\left(2 c_{W}\right) \bar{u}_{p_{1}} \gamma_{\lambda}\left(g_{v}^{\ell^{\prime}}-g_{a}^{\ell^{\prime}} \gamma_{5}\right) v_{p_{2}}{ }^{2}$ is independent of the loop integration, whereas the effective $Z L \ell$ transition is given as follows:

$l_{L \ell}^{\lambda}=\left(\frac{-i g}{4 c_{W}}\right)\left(\frac{-i g}{2 \sqrt{2}}\right)^{2} \sum_{j=1}^{3} U_{\ell j}^{*} U_{L j} \bar{u}_{p} \Gamma_{j}^{\lambda} u_{P}$,

where $U_{i m}$ are entries of the PMNS mixing matrix. In the Feynman-'t Hooft gauge, we have

$\Gamma_{j}^{\lambda}=\int \frac{d^{4} k}{(2 \pi)^{4}} \frac{N^{\lambda}}{\left[(p+k)^{2}-m_{j}^{2}\right]\left[(P+k)^{2}-m_{j}^{2}\right]\left[k^{2}-m_{W}^{2}\right]}$

with

$$
\begin{aligned}
N^{\lambda}= & \gamma_{\rho}\left(1-\gamma_{5}\right) i\left[(\not p+\not k)+m_{j}\right] \gamma^{\lambda}\left(1-\gamma_{5}\right) i\left[(\not p+\not k)+m_{j}\right] \\
& \times \gamma_{\sigma}\left(1-\gamma_{5}\right)\left(-i g^{\rho \sigma}\right) .
\end{aligned}
$$

After making the loop integration using dimensional regularization in order to deal with the (logarithmic) UV divergences, the Lorentz structure for the $\Gamma_{j}^{\lambda}$ factor can be written as follows,

$$
\begin{aligned}
\Gamma_{j}^{\lambda}= & F_{a} \gamma^{\lambda}\left(1-\gamma_{5}\right)+F_{b} \gamma^{\lambda}\left(1+\gamma_{5}\right) \\
& +F_{c}(P+p)^{\lambda}\left(1+\gamma_{5}\right)+F_{d}(P+p)^{\lambda}\left(1-\gamma_{5}\right) \\
& +F_{e} q^{\lambda}\left(1+\gamma_{5}\right)+F_{f} q^{\lambda}\left(1-\gamma_{5}\right),
\end{aligned}
$$

where in general $F_{k}=F_{k}\left(q^{2}, m_{j}^{2}\right)(k=a, b \ldots, f)$ are functions given in terms of the momentum transfer $q^{2}=(P-p)^{2}$, and the neutrino mass squared (of course $F_{k}$ functions will also depend on the mass of the $W$ gauge boson and external masses, but these have well-defined values).

At this point, it is worth to note that in the approximation where the momenta of the external particles are neglected in Eq. (3), such as it is done in Ref. [15] for the $\mu \rightarrow 3 e$ decay, the computation is simplified considerably, as the only possible contribution is given by the $F_{a}^{0}$ function, where we are using a superscript 0 in order to distinguish this approximation. In this simple case, the $F_{a}^{0}$ function will not depend

\footnotetext{
$2 g$ is the $S U(2)_{L}$ coupling and $c_{W}\left(s_{W}\right)$ is short for the cosine(sine) of the weak mixing angle $\theta_{W}$. In the SM, $g_{v}^{\ell^{\prime}}=-1 / 2+2 s_{W}^{2}$ and $g_{a}^{\ell^{\prime}}=-1 / 2$.
} 


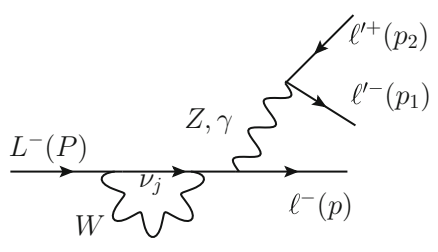

(a)

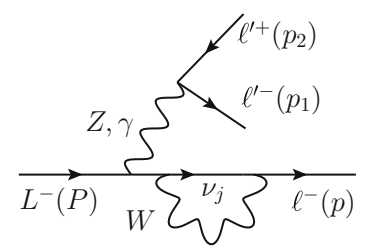

(b)

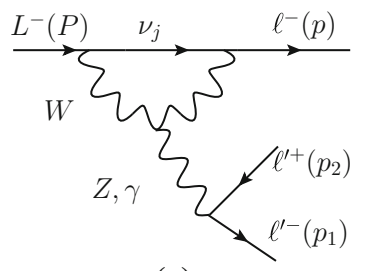

(c)

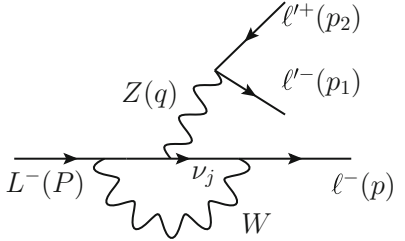

(d)

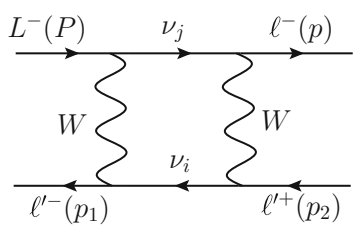

(e)
Fig. 1 Feynman diagrams for the $L^{-} \rightarrow \ell^{-} \ell^{\prime-} \ell^{\prime+}$ decays in the presence of lepton mixing (i.e., non-vanishing neutrino masses). In 'renormalizable' $R_{\xi}$ gauges, similar diagrams need to be added, which are obtained replacing the $W$ gauge bosons by the respective would-be Goldstone bosons. Notice that diagram $\mathbf{d}$ only involves the $Z$ gauge boson, whereas the $\mathbf{a}, \mathbf{b}$ and $\mathbf{c}$ diagrams can also be mediated by the

on $q^{2}$ and is given in terms of the Feynman parameters as follows

$F_{a}^{0}\left(m_{j}^{2}\right)=\frac{1}{2 \pi^{2}} \int_{0}^{1} d x \int_{0}^{1-x}\left[2+\log \left(D_{j}^{0} / \mu^{2}\right)\right] d y$,

where $D_{j}^{0}\left(m_{j}^{2}\right)=(1-x) m_{j}^{2}+x m_{W}^{2}$. Whereas in terms of $\mathrm{PaVe}$ functions it is given by

$$
\begin{aligned}
F_{a}^{0}\left(m_{j}^{2}\right)= & -\frac{1}{8 \pi^{2}\left(m_{j}^{2}-m_{W}^{2}\right)^{2}}\left[2 m_{j}^{2}\left(m_{j}^{2}-2 m_{W}^{2}\right)\right. \\
& \times B_{0}\left(0, m_{j}^{2}, m_{j}^{2}\right)+2 m_{W}^{4} B_{0}\left(0, m_{W}^{2}, m_{W}^{2}\right) \\
& \left.+4 m_{j}^{2} m_{W}^{2}-3 m_{j}^{4}-m_{W}^{4}\right] .
\end{aligned}
$$

Now, one analytical expression for the $F_{a}^{0}$ function can be obtained in a straightforward way either integrating over the Feynman parameters in Eq. (6) or using the definition of the $B_{0}\left(0, m^{2}, m^{2}\right)$ scalar function in Eq. (7). In such a way that after making an expansion around $m_{j}^{2}=0$ we obtained

$$
\begin{aligned}
F_{a}^{0}= & \frac{1}{2 \pi^{2}}\left[\frac{m_{j}^{2}}{m_{W}^{2}} \log \left(\frac{m_{W}^{2}}{m_{j}^{2}}\right)-\frac{m_{j}^{2}}{2 m_{W}^{2}}+\frac{1}{2} \log \left(\frac{m_{W}^{2}}{\mu^{2}}\right)\right. \\
& \left.+\frac{1}{4}+\vartheta\left(\frac{m_{j}^{2}}{m_{W}^{2}}\right)^{2}\right] .
\end{aligned}
$$

From Eq. (8) it becomes clear that, in this approximation, the amplitude will be proportional to the neutrino mass squared, where the dominant contribution, due to the big gap photon. Additionally, when $\ell=\ell^{\prime}$ similar contributions (exchanging $p \leftrightarrow p_{1}$ ) to the amplitudes of diagrams a-e must be subtracted in order to antisymmetrize the amplitude. On the other hand, when $\ell \neq \ell^{\prime}$, since the vertices of the neutral bosons $\gamma$ and $Z$ with a pair of fermions are flavor-conserving, only a similar e box diagram must be added interchanging $\ell(p) \leftrightarrow \ell^{\prime}\left(p_{1}\right)$

between the neutrino and $W$ boson mass scales, comes from the first term as it involves a relative factor $\log \left(\frac{m_{W}^{2}}{m_{j}^{2}}\right)$ compared to the second one, ${ }^{3}$ whereas the terms independent of neutrino masses will vanish by the GIM-like mechanism.

Therefore, the structure of the matrix element for the contribution of the diagram (d) in Fig. 1 in the approximation where masses and momenta of the external particles are neglected is given by

$$
\begin{aligned}
\mathscr{M}_{d}^{0}= & -i \frac{G_{F}^{2} m_{W}^{2} \beta_{F_{a}^{0}}}{4} \bar{u}_{p} \gamma_{\lambda}\left(1-\gamma_{5}\right) u_{P} \times \bar{u}_{p_{1}} \gamma^{\lambda}\left(1-\gamma_{5}\right) v_{p_{2}} \\
& +i G_{F}^{2} m_{W}^{2} s_{W}^{2} \beta_{F_{a}^{0}} \bar{u}_{p} \gamma_{\lambda}\left(1-\gamma_{5}\right) u(P) \times \bar{u}_{p_{1}} \gamma^{\lambda} v_{p_{2}},
\end{aligned}
$$

where we have defined

$\beta_{F_{a}^{0}}=\sum_{j} U_{L j} U_{\ell j}^{*} F_{a}^{0}\left(m_{j}^{2}\right)$

We verified that Eq. (9) reproduces the result reported in Ref. [15] considering only the first term in Eq. (8) and the simple case of two families.

Returning to the general case (non-zero masses and momenta of the external particles), we also have obtained the $F_{k}$ functions using both Feynman parametrization (we will denote the corresponding expressions by $F_{F_{k}}$ ) and the Passarino-Veltman (PaVe) technique (denoted by $F_{P V_{k}}$ ) [27,28], employing FeynCalc [29]. In particular, we agree

\footnotetext{
3 A similar relative suppression operates for the diagrams in Fig. 1a-c with respect to the diagrams in Fig. 1d, e.
} 
with the expressions previously reported in Ref. [17] in terms of the Feynman parameters, ${ }^{4}$ namely the $F_{F_{k}}$ functions can be written as

$F_{F_{k}}\left(q^{2}, m_{j}^{2}\right)=\frac{1}{2 \pi^{2}} \int_{0}^{1} d x \int_{0}^{1-x} f_{k}\left(q^{2}, m_{j}^{2}\right) d y$,

where

$$
\begin{aligned}
f_{a}= & 2+\log \left(D_{j}\left(q^{2}\right) / \mu^{2}\right) \\
& +\frac{\left(q^{2}-m^{2}\right) x(y-1)+M^{2} x(x+y)+q^{2} y(y-1)}{D_{j}},
\end{aligned}
$$

$f_{b}=\frac{m M x}{D_{j}}$,

$f_{c}=-\frac{M x(x+y)}{D_{j}}$,

$f_{d}=-\frac{m x(1-y)}{D_{j}}$,

$f_{e}=\frac{M x(2-3 y-x)-2 M y(y-1)}{D_{j}}$,

$f_{f}=\frac{x m(y-1)+2 m y(y-1)}{D_{j}}$,

and $D_{j}$ is defined as

$$
\begin{aligned}
D_{j}\left(q^{2}, m_{j}^{2}\right)= & -(x-1) m_{j}^{2}-m^{2} x y+x m_{W}^{2} \\
& +M^{2} x(x+y-1)-q^{2} y(1-x-y) .
\end{aligned}
$$

We have omitted in $f_{a}$ the term associated with the UV divergence since it is independent of $m_{j}$ and vanishes owing to the GIM-like mechanism.

On the other hand, the $F_{k}$ functions in terms of the PaVe scalar functions are given as follows

$F_{P V_{k}}\left(q^{2}, m_{j}^{2}\right)=\frac{1}{2 \pi^{2}} \frac{N_{F_{k}}}{D_{F_{k}}}$,

with

$$
\begin{aligned}
D_{F_{a}}= & 2 D_{F_{b}}=-2 \lambda\left(m^{2}, M^{2}, q^{2}\right), \\
D_{F_{c}}= & D_{F_{e}}=\frac{M}{2} D_{F_{a}}^{2} \quad D_{F_{d}}=D_{F_{f}}=\frac{m}{2} D_{F_{a}}^{2}, \\
N_{F_{k}}= & \xi_{k_{1}} B_{0}\left(m^{2}, m_{j}^{2}, m_{W}^{2}\right)+\xi_{k_{2}} B_{0}\left(M^{2}, m_{j}^{2}, m_{W}^{2}\right) \\
& +\xi_{k_{3}} B_{0}\left(q^{2}, m_{j}^{2}, m_{j}^{2}\right)+\xi_{k_{4}} B_{0}\left(0, m_{j}^{2}, m_{W}^{2}\right) \\
& +\xi_{k_{5}} C_{0}\left(m^{2}, M^{2}, q^{2}, m_{j}^{2}, m_{W}^{2}, m_{j}^{2}\right)+\xi_{k_{0}},
\end{aligned}
$$

\footnotetext{
${ }^{4}$ We have found some irrelevant differences in the numerators of the $f_{d}$ and $f_{f}$ functions, as can be seen comparing Eqs. (15) and (17) with the corresponding expressions in Ref. [17].
}

where $\lambda$ is the Kallen function $\lambda(x, y, z)=x^{2}+y^{2}+z^{2}-$ $2(x y+x z+y z)$, and the $\xi_{k}$ factors can be found in the "Appendix A". 5

Unlike the approximation made in Ref. [15], the presence of masses and momenta of the external particles in the computation hinders the way for the derivation of analytical expressions for the integrals in Eqs. (11) or (19). ${ }^{6}$ Nevertheless, we have done a numerical cross-check between both expressions, where we have employed the Looptools package $[30,31]$ for the evaluation of the PaVe functions and a numerical Mathematica [32] routine for the evaluation of the parametric integrals (see Fig. 2). We have found an excellent agreement between these two expressions for values of $q^{2}<4 m_{j}^{2}$, which are, however, outside of the physical domain for the considered decays, since $q_{\min }^{2}=4 m_{\ell^{\prime}}^{2} \gg m_{j}^{2}$. In this way, owing to the simplified integrals, we verified that a better precision is found in terms of $\mathrm{PaVe}$ functions than using Feynman parameters. This feature is illustrated, as an example, for the particular case of the $Z \tau \mu$ transition in Fig. 2 for the (dominant, as we will show) $F_{a}$ factor.

At this point, we want to stress that we disagree with the approximation done in Ref. [17] in order to estimate the relevant dependence on the neutrino mass of the $F_{k}$ functions. We highlight that we are studying a process where the momentum transfer $q^{2}$ must be non-vanishing and in principle is much larger than the neutrino squared mass, $m_{j}^{2}$, which comes from the loop computation. Therefore, using an expansion around $q^{2}=0$ in order to simplify the integration over the Feynman parameters keeping the terms proportional to $m_{j}^{2}$ in the denominators of Eqs. (12-17), as it is done in Ref. [17], modifies substantially the behavior of the original functions in the interesting physical region for the neutrino masses and, as a consequence, it gives rise to an incorrect infrared logarithmically divergent behavior of the $F_{k}$ functions when $m_{j}$ goes to zero, without any possible cure. In particular, the dependence on the momentum transfer, $q^{2}$, plays a crucial role in the behavior of the $F_{k}$ functions. In this respect, we point out the presence of a small imaginary part in the $F_{a}$ function, which emerges for the physical values $4 m_{j}^{2}<q^{2}$.

As we mentioned before, the $q^{2}$ minimum in the $L^{-} \rightarrow$ $\ell^{-} \ell^{\prime}-\ell^{\prime+}$ decay is given by $4 m_{\ell^{\prime}}^{2}$, which is much larger than the neutrinos masses. This, together with the difficulties in obtaining analytical expressions directly for the $F_{k}$ functions

\footnotetext{
5 The cancellation of the UV divergences for the $F_{m}$ functions in terms of the PaVe functions occurs again by the GIM mechanism. This can be verified easily owing to the fact that the sums over the coefficients of the different scalar $B_{0}$ functions, which contain an isolated divergent term, are independent of $m_{j}$. That is $\sum_{i=1}^{4} \frac{\xi_{a_{i}}}{D_{P V_{a}}}=-\frac{1}{2}$, and $\sum_{i=1}^{4} \frac{\xi_{l_{i}}}{D_{P V_{l}}}=0$ for $(l=b, c, d, e, f)$.

6 The analytical expressions of the first integrals over the $y$ parameter in Eqs. (12-17) can be derived from the formulas reported in "Appendix B".
} 


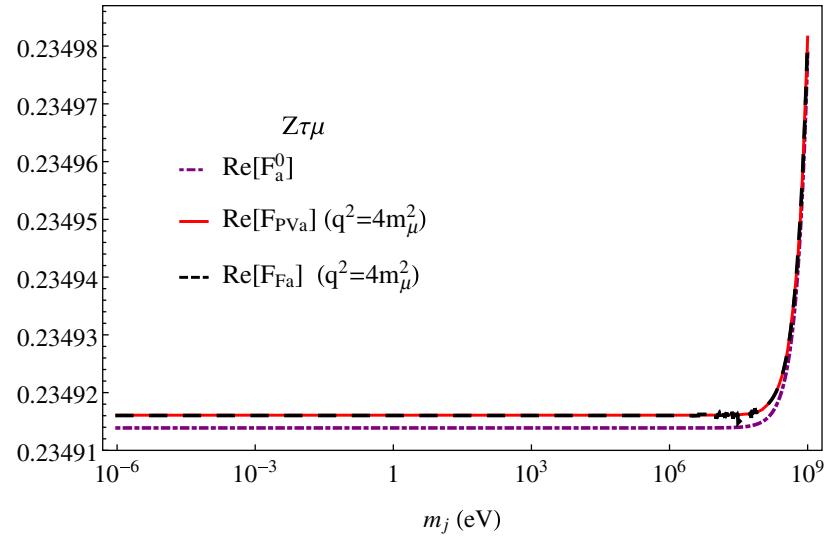

Fig. 2 Numerical evaluation of the $F_{a}$ function for the effective $Z \tau \mu$ vertex as a function of the neutrino mass, taking the minimal value of $q^{2}=4 m_{\mu}^{2}$ for the particular $\tau^{-} \rightarrow \mu^{-} \mu^{-} \mu^{+}$channel. Black dashed line stands for the numerical evaluation of the $F_{a}$ function in terms of the Feynman parameters depicted by $F_{F_{a}}$ (Eq. (11)), whereas the red line corresponds to the evaluation in terms of the PaVe functions represented by $F_{P V_{a}}$ (Eq. (19)). We have found some numerical instabilities for the evaluation of the $F_{F_{a}}$ function in the region $0.01 \mathrm{GeV}<m_{j}<0.1$ $\mathrm{GeV}$. On the other hand, better precision is achieved in the evaluation of the $F_{P V_{a}}$ function with the help of the Looptools package. In order to perform a comparison with the approximation done in Ref. [15], we also show the complete $F_{a}^{0}$ given by the Eqs. (6) or (7) (purple dotdashed line)

suggests employing some numerical approximation to deal with the problem. Because of this, we approximate the $F_{k}$ functions in the physical region for the neutrinos masses by fitting the curves for the real and imaginary parts of the $F_{k}$ functions evaluated in terms of the PaVe function ${ }^{7}$ We have found a reasonably good fit of the form

$F_{k}=\frac{1}{2 \pi^{2} u}\left(Q_{k}+\frac{m_{j}^{2}}{m_{W}^{2}} R_{k}\right)$,

where $u=1$ for $k=a, b$ and $u=M$ for $k=c, d, e, f$ and the respective values for the $Q_{k}=Q_{R_{k}}+i Q_{R_{I}}$ and $R_{k}=R_{R_{k}}+i R_{R_{I}}$ factors of all considered channels are given in "Appendix D".

From Eq. (23), it turns clear that the $Q_{k}$ factors will not contribute owing to the GIM-like mechanism, whereas the relevant contributions are given by the $R_{k}$ factors. Then, according to our numerical results, we find that the relevant factors of the $F_{b}, F_{c}$ and $F_{d}$ functions are suppressed with respect to the $F_{a}$ factor. On the other hand, despite the respective factors of $F_{e}$ and $F_{f}$ functions are larger than those of the $F_{a}$ function, when the momentum transfer becomes smaller and smaller their helicity suppression makes them negligible.

\footnotetext{
${ }^{7}$ Our fits for the $F_{k}$ functions are taken with the precision of the Looptools package considering a neutrino mass varying from $10^{-15} \mathrm{GeV}$ to the benchmark point $m_{\mu}\left(m_{e}\right)$, for a fixed value of $q^{2}=4 m_{\mu}^{2}$ $\left(q^{2}=4 m_{e}^{2}\right)$ for the $Z \tau \mu(Z \tau e$ and $Z \mu e)$ vertices.
}

Therefore, we will concentrate on the contribution of the $F_{a}$ function.

Furthermore, in order to check our results, we have made an expansion for the PaVe functions involved in Eq. (22), following the same strategy that Cheng and Li for the $\mu \rightarrow e \gamma$ decay [13], that is: expanding the loop integrals around $m_{j}^{2}=$ 0 (more details on our expansions are given in "Appendix E"). It must be noted that, since neutrino masses are the smallest energy scale in the problem, this is the expansion that is most efficient for the considered decays. Using the PackageX program [35], we could rewrite the $F_{P V_{a}}$ contribution as follows:

$F_{P V_{a}}\left(q^{2}, m_{j}^{2}\right)=\frac{1}{2 \pi^{2}}\left[Q_{a}+\frac{m_{j}^{2}}{m_{W}^{2}} R_{a}+\vartheta\left(\frac{m_{j}^{4}}{m_{W}^{4}}\right)\right]$,

where

$$
\begin{aligned}
Q_{a}= & -\lambda\left(m^{2}, M^{2}, q^{2}\right)^{-1}\left[f_{Q_{a_{1}}} C_{0}\left(m^{2}, M^{2}, q^{2}, 0, m_{W}^{2}, 0\right)\right. \\
& +f_{Q_{a_{2}}} \log \left(\frac{m_{W}^{2}}{m_{W}^{2}-m^{2}}\right)+f_{Q_{a_{3}}} \log \left(\frac{m_{W}^{2}}{m_{W}^{2}-M^{2}}\right) \\
& \left.+f_{Q_{a_{4}}} \log \left(\frac{m_{W}^{2}}{q^{2}}\right)+f_{Q_{a_{5}}}\right]-\frac{1}{2} \Delta, \\
R_{a}= & -m_{W}^{2} \lambda\left(m^{2}, M^{2}, q^{2}\right)^{-1}\left[f_{R_{a_{1}}} C_{0}\left(m^{2}, M^{2}, q^{2}, 0, m_{W}^{2}, 0\right)\right. \\
& +f_{R_{a_{2}}} \log \left(\frac{m_{W}^{2}}{m_{W}^{2}-m^{2}}\right)+f_{R_{a_{3}}} \log \left(\frac{m_{W}^{2}}{m_{W}^{2}-M^{2}}\right) \\
& \left.+f_{R_{a_{4}}} \log \left(\frac{m_{W}^{2}}{q^{2}}\right)+f_{R_{a_{5}}}\right],
\end{aligned}
$$

in which $\Delta=\frac{1}{\epsilon}-\gamma_{E}+\log (4 \pi)$, and the $f_{Q}$ and $f_{R}$ factors can be found in the "Appendix E". We verified that our numerical fits for the $Z \tau \mu$ and $Z \tau e$ vertex are in a very good agreement with Eq. (26), whereas a deviation is found for the $Z \mu e$ vertex, as can be seen in Table 9. We consider the results obtained from Eq. (26) for the effective vertices as our reference ones.

In this way, we can approximate the amplitude for the diagram (d) according to Eq. (9) replacing $F_{a}^{0}$ by

$F_{a} \approx \frac{1}{2 \pi^{2}} \frac{m_{j}^{2}}{m_{W}^{2}} R_{a}$

The penguin contributions to the branching ratios of the $L^{-} \rightarrow \ell^{-} \ell^{\prime-} \ell^{\prime+}$ decays are smaller by around one (two) orders of magnitude for the $\mu(\tau)$ decays when one keeps external momenta and masses than when they are neglected. We will see in Sect. 4 a similar difference in the full result. 


\section{Contributions of the box diagrams}

Now, in order to make a complete comparison with the approximation done in Ref. [15] we have also obtained the amplitude for the box diagram (e) in Fig. 1. Note that unlike the penguin diagram (d), which involves two neutrino propagators of the same mass state, the box diagram (e) can involve two neutrino propagators with different mass states. Thus, in full generality, the amplitude can be written as follows:

$\mathscr{M}_{e}=\left(\frac{-i g}{2 \sqrt{2}}\right)^{4} \sum_{i, j} U_{L j} U_{l j}^{*} U_{\ell^{\prime} i} U_{\ell^{\prime} i}^{*} T_{\sigma \sigma^{\prime}} I^{\sigma \sigma^{\prime}}$,

where we defined

$T_{\sigma \sigma^{\prime}}=4 \bar{u}_{p} \gamma_{\mu} \gamma_{\sigma} \gamma_{\nu}\left(1-\gamma_{5}\right) u_{P} \times \bar{u}_{p_{1}} \gamma^{\nu} \gamma_{\sigma^{\prime}} \gamma^{\mu}\left(1-\gamma_{5}\right) v_{p_{2}}$,

and the relevant loop integral is given by (see Fig. 1e)

$I^{\sigma \sigma^{\prime}}=\int \frac{d^{4} k}{(2 \pi)^{4}} \frac{(P+k)^{\sigma}\left(k+p_{1}\right)^{\sigma^{\prime}}}{D_{B}}$,

with

$$
\begin{aligned}
D_{B}= & \left(k^{2}-m_{W}^{2}\right)\left[\left(p_{1}+p_{2}+k\right)^{2}-m_{W}^{2}\right]\left[(P+k)^{2}-m_{j}^{2}\right] \\
& \times\left[\left(k+p_{1}\right)^{2}-m_{i}^{2}\right] .
\end{aligned}
$$

Since we have written the Eq. (30) in terms of the momenta $P, p_{1}$ and $p_{2}$, the integral must take the form

$$
\begin{aligned}
I^{\sigma \sigma^{\prime}}= & i\left(g^{\sigma \sigma^{\prime}} H_{a}+P^{\sigma} P^{\sigma^{\prime}} H_{b}+P^{\sigma} p_{1}^{\sigma^{\prime}} H_{c}+P^{\sigma} p_{2}^{\sigma^{\prime}} H_{d}\right. \\
& +p_{1}^{\sigma} P^{\sigma^{\prime}} H_{e}+p_{1}^{\sigma} p_{1}^{\sigma^{\prime}} H_{f}+p_{1}^{\sigma} p_{2}^{\sigma^{\prime}} H_{g}+p_{2}^{\sigma} P^{\sigma^{\prime}} H_{h} \\
& \left.+p_{2}^{\sigma} p_{1}^{\sigma^{\prime}} H_{i}+p_{2}^{\sigma} p_{2}^{\sigma^{\prime}} H_{j}\right) .
\end{aligned}
$$

The $H_{k}$ factors depend upon the kinematic variables $s_{12}=$ $\left(p_{1}+p_{2}\right)^{2}=q^{2}$ and $s_{13}=\left(p_{1}+p\right)^{2}$, in addition of $m_{i}$ and $m_{j}$.

Anew, in the approximation where momenta of the external particles are neglected in Eq. (30), the only contribution is given by the $H_{a}^{0}$ function, which will not depend either on $s_{12}$ or $s_{13}$. In such a case, we obtained the following simplified expression

$H_{a}^{0}\left(m_{j}^{2}, m_{i}^{2}\right)=\frac{1}{16 \pi^{2}} \int_{0}^{1} d x \int_{0}^{1-x} d y \int_{0}^{1-x-y} \frac{-1}{2 M_{F_{0}}^{2}} d z$

where

$M_{F_{0}}^{2}=m_{W}^{2}(x+y)-m_{j}^{2}(x+y-1)+\left(m_{i}^{2}-m_{j}^{2}\right) z$.
Whereas, in terms of PaVe functions, $H_{a}^{0}$ reads

$$
\begin{aligned}
H_{a}^{0}\left(m_{j}^{2}, m_{i}^{2}\right) & \\
= & \frac{1}{16 \pi^{2}}\left(\frac{m_{j}^{4}}{4\left(m_{j}^{2}-m_{i}^{2}\right)\left(m_{j}^{2}-m_{W}^{2}\right)^{2}} B_{0}\left(0, m_{j}^{2}, m_{j}^{2}\right)\right. \\
& +\frac{m_{i}^{4}}{4\left(m_{i}^{2}-m_{j}^{2}\right)\left(m_{i}^{2}-m_{W}^{2}\right)^{2}} B_{0}\left(0, m_{i}^{2}, m_{i}^{2}\right) \\
& +\frac{2 m_{i}^{2} m_{j}^{2} m_{W}^{2}-m_{W}^{4}\left(m_{i}^{2}+m_{j}^{2}\right)}{4\left(m_{i}^{2}-m_{W}^{2}\right)^{2}\left(m_{j}^{2}-m_{W}^{2}\right)^{2}} B_{0}\left(0, m_{W}^{2}, m_{W}^{2}\right) \\
& \left.+\frac{m_{W}^{2}}{4\left(m_{i}^{2}-m_{W}^{2}\right)\left(m_{W}^{2}-m_{j}^{2}\right)}\right)
\end{aligned}
$$

In the same way as for the $F_{a}^{0}$ form factor, an analytical expression for $H_{a}^{0}$ can be obtained easily from either Eqs. (33) or (35). This time, making a double Taylor expansion, first around $m_{i}^{2}=0$ and then around $m_{j}^{2}=0$, we obtained that

$$
\begin{aligned}
H_{a}^{0}\left(m_{j}^{2}, m_{i}^{2}\right)= & \frac{1}{64 \pi^{2} m_{W}^{4}}\left[\left(m_{i}^{2}+m_{j}^{2}\right)\left(\log \left(\frac{m_{W}^{2}}{m_{j}^{2}}\right)-1\right)\right. \\
& +\frac{m_{i}^{2} m_{j}^{2}}{m_{W}^{2}}\left(2 \log \left(\frac{m_{W}^{2}}{m_{j}^{2}}\right)-1\right) \\
& \left.+-m_{W}^{2}+\vartheta\left(\frac{m_{i}^{4}}{m_{W}^{2}}\right) \vartheta\left(\frac{m_{j}^{4}}{m_{W}^{2}}\right)\right] .
\end{aligned}
$$

Using that $T_{\sigma \sigma^{\prime}} g^{\sigma \sigma^{\prime}}=16 \bar{u}_{p} \gamma_{\lambda}\left(1-\gamma_{5}\right) u_{P} \times \bar{u}_{p_{1}} \gamma^{\lambda}(1-$ $\left.\gamma_{5}\right) v_{p_{2}}$, the amplitude -in this approximation- is given by

$\mathscr{M}_{e}^{0}=i 8 G_{F}^{2} m_{W}^{4} \beta_{H_{a}^{0}} \bar{u}_{p} \gamma_{\lambda}\left(1-\gamma_{5}\right) u_{P} \times \bar{u}_{p_{1}} \gamma^{\lambda}\left(1-\gamma_{5}\right) v_{p_{2}}$,

with

$\beta_{H_{a}^{0}}=\sum_{j, i} U_{L j} U_{\ell j}^{*} U_{\ell^{\prime} i} U_{\ell^{\prime} i}^{*} H_{a}^{0}\left(m_{i}^{2}, m_{j}^{2}\right)$.

Again, we verified that taking into account the first term in Eq. (36) and considering only two families, Eq. (37) reproduces the expression reported in Ref. [15] for the amplitude of the box diagram 1 (e) in the $\mu \rightarrow 3 e$ decay. Furthermore, our results are consistent with the previous expressions reported in Ref. [23] for the box contribution associated with the effective $K^{+} \rightarrow \pi^{+} v_{\ell} \bar{\nu}_{\ell}$ decay in the quark sector, where the approximation of taking masses and momenta of the external particles equal to zero is excellent, owing to the presence of the heavy top quark inside the loop. 
In the general case, we also obtained the $H_{k}(k=$ $a, b, \ldots, j)$ functions in terms of both Feynman parameters integrals, $H_{F_{k}}$, and PaVe functions, $H_{P V_{k}}$. This time, the $H_{k}$ functions will depend on the squared masses of two different neutrinos, $m_{j}^{2}$ and $m_{i}^{2}$, and on two independent phase space variables $s_{12}$ and $s_{13}$. Using Feynman parametrization these functions read

$$
\begin{aligned}
& H_{F_{k}}\left(s_{12}, s_{13}, m_{i}^{2}, m_{j}^{2}\right) \\
& =\frac{1}{16 \pi^{2}} \int_{0}^{1} d x \int_{0}^{1-x} d y \int_{0}^{1-x-y} h_{k} d z,
\end{aligned}
$$

where

$$
\begin{aligned}
h_{a} & =-\frac{1}{2 M_{F}^{2}}, \\
h_{b} & =\frac{z(z-1)}{M_{F}^{4}}, \\
h_{c} & =-\frac{(z-1)(x+z)}{M_{F}^{4}}, \\
h_{d} & =\frac{y(z-1)}{M_{F}^{4}}, \\
h_{e} & =-\frac{z(x+z-1)}{M_{F}^{4}}, \\
h_{f} & =\frac{(x+z-1)(x+z)}{M_{F}^{4}}, \\
h_{g} & =-\frac{y(x+z-1)}{M_{F}^{4}}, \\
h_{h} & =\frac{y z}{M_{F}^{4}}, \\
h_{i} & =-\frac{y(x+z)}{M_{F}^{4}}, \\
h_{j} & =\frac{y^{2}}{M_{F}^{4}} \cdot
\end{aligned}
$$

In the previous expressions, the denominator function is given by

$$
\begin{aligned}
M_{F}^{2}= & -m_{j}^{2}(x+y-1)+m_{\ell^{\prime}}^{2}(x+y-1)(x+y) \\
& +m_{W}^{2}(x+y) \\
& -s_{12} x y+z^{2}\left(2 m_{\ell^{\prime}}^{2}+m^{2}+M^{2}-s_{12}-s_{13}\right) \\
& +z\left[m_{i}^{2}-m_{j}^{2}+(x+y)\left(3 m_{\ell^{\prime}}^{2}-s_{12}-s_{13}\right)-2 m_{\ell^{\prime}}^{2}\right. \\
& \left.+m^{2}(x-1)+M^{2}(y-1)+s_{12}+s_{13}\right] .
\end{aligned}
$$

Expressions are rather lengthy in terms of the PaVe functions so that here we only present the expression for the dominant $H_{a}$ function, which can be written as

$$
H_{P V_{a}}\left(s_{12}, s_{13}, m_{j}^{2}, m_{i}^{2}\right)=\frac{1}{16 \pi^{2}} \frac{N_{H_{a}}}{D_{H_{a}}},
$$

with

$$
\begin{aligned}
D_{H_{a}}= & 4\left(m^{4} m_{\ell^{\prime}}^{2}-m^{2}\left[M^{2}\left(2 m_{\ell^{\prime}}^{2}-s_{12}\right)+s_{12}\left(m_{\ell^{\prime}}^{2}+s_{13}\right)\right]\right. \\
& +M^{4} m_{\ell^{\prime}}^{2}-M^{2} s_{12}\left(m_{\ell^{\prime}}^{2}+s_{13}\right) \\
& \left.+s_{12}\left(-2 s_{13} m_{\ell^{\prime}}^{2}+m_{\ell^{\prime}}^{4}+s_{13}\left(s_{12}+s_{13}\right)\right)\right)
\end{aligned}
$$

and

$$
\begin{aligned}
N_{H_{a}}= & \chi_{k_{1}} C_{0}\left(m^{2}, M^{2}, s_{12}, m_{W}^{2}, m_{i}^{2}, m_{W}^{2}\right) \\
& +\chi_{k_{2}} C_{0}\left(m_{\ell^{\prime}}^{2}, m_{\ell^{\prime}}^{2}, s_{12}, m_{W}^{2}, m_{j}^{2}, m_{W}^{2}\right) \\
& +\chi_{k_{3}} C_{0}\left(M^{2}, m_{\ell^{\prime}}^{2}, m^{2}+M^{2}\right. \\
& \left.+2 m_{\ell^{\prime}}^{2}-s_{12}-s_{13}, m_{i}^{2}, m_{W}^{2}, m_{j}^{2}\right) \\
& +\chi_{k_{4}} C_{0}\left(m^{2}, m_{\ell^{\prime}}^{2}, m^{2}+M^{2}\right. \\
& \left.+2 m_{\ell^{\prime}}^{2}-s_{12}-s_{13}, m_{i}^{2}, m_{W}^{2}, m_{j}^{2}\right) \\
& +\chi_{k_{5}} D_{0}\left(m^{2}, M^{2}, m_{\ell^{\prime}}^{2}, m_{\ell^{\prime}}^{2}, s_{12}, m^{2}\right. \\
& +M^{2}+2 m_{\ell^{\prime}}^{2}-s_{12} \\
& \left.-s_{13}, m_{W}^{2}, m_{i}^{2}, m_{W}^{2}, m_{j}^{2}\right)
\end{aligned}
$$

where $\chi_{k}$ factors are reported in "Appendix A".

As far as the general case is concerned, we can see that although there are additional contributions associated with the $H_{k}$ functions, with $k=b, c, d, \ldots j$; they are expected to be suppressed, as they correspond to higher-dimensional operators, with respect to the $H_{a}$ function associated with a $(V-A) \times(V-A)$ operator. Therefore, we will concentrate on the $H_{a}$ function in order to estimate the box diagram contribution. We also have done a numerical cross-check between the expressions for the $H_{a}$ function given in terms of the Feynman parameters Eq. (39) and the PaVe functions Eq. (51), as can be seen in Fig. 3. In this case, it turns very complicated and far away of the purpose of this work to obtain an analytical expression for the $H_{a}$ function in Eq. (53) making an expansion for the respective scalar PaVe functions, owing to the number of propagators involved and the dependence on two different neutrino masses. However, we can expect a good approximation through our numerical results, as it happens with the penguin contribution.

Thus, we estimate the relevant dependence on the neutrino mass for the $H_{a}$ function fitting the curve for the real and imaginary parts of the $H_{a}$ function evaluated in terms of the $\mathrm{PaVe}$ functions considering fixed values for the $m_{i}, s_{12}$, and 


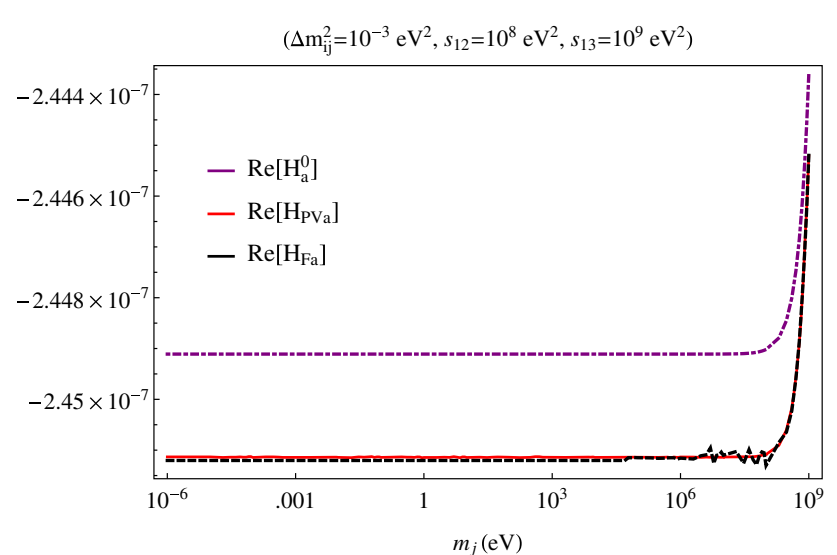

Fig. 3 Numerical evaluation of the $H_{a}$ function versus the neutrino mass. We are considering that $\Delta m_{i j}^{2}=10^{-3} \mathrm{eV}^{2}$ and the values of $s_{12}=10^{8} \mathrm{eV}^{2}$ and $s_{13}=10^{9} \mathrm{eV}^{2}$ associated with a representative point in the physical phase space for the particular $\tau^{-} \rightarrow \mu^{-} \mu^{-} \mu^{+}$ channel. In analogous way to the Fig. 2, black dashed line stands for the numerical evaluation of the $H_{a}$ function in terms of the Feynman parameters depicted by $H_{F_{a}}$ (Eq. (39)), whereas the red line corresponds to the evaluation in terms of the PaVe functions represented by $H_{P V_{a}}$ (Eq. (51)). Numerical instabilities for the evaluation of the $H_{F_{a}}$ function around $0.001 \mathrm{GeV}<m_{j}<1 \mathrm{GeV}$ are found. A better precision is achieved for the evaluation of the $F_{P V_{a}}$ function with the help of the Looptools package. In order to perform a comparison with the approximation done in Ref. [15], we also show the complete $H_{a}^{0}$ given by the Eqs. (33) or (35) (purple dotdashed line)

$s_{13}$ parameters. $^{8}$ We obtained a good fit of the form

$H_{a}=\frac{1}{16 \pi^{2}}\left(Q_{H_{a}}+\frac{m_{j}^{2}}{m_{W}^{4}} R_{H_{a}}\right)$,

where $R_{H_{a}} \approx 1.5+i 0.007$, for all different $\tau \rightarrow \ell^{-} \ell^{\prime-} \ell^{\prime+}$ channels, whereas $R_{H_{a}} \approx 1.5$, for the $\mu^{-} \rightarrow e^{-} e^{-} e^{+}$channel. These numbers were obtained considering that $\Delta m_{i j}^{2}=$ $10^{-3} \mathrm{eV}^{2}$, and representative values for $s_{12}$ and $s_{13}$ within the corresponding phase space.

\section{Numerical results}

In order to evaluate the branching fractions for the $L^{-} \rightarrow$ $\ell^{-} \ell^{\prime-} \ell^{\prime+}$ decays we consider the results of the previous sections and the state of the art best-fit values of the three neutrino oscillation parameters [18-21]. Without loss of generality, we assume the $C P$-conserving scenario, and we use the values reported in Table $1 .{ }^{9}$ The kinematics for the $L^{-} \rightarrow \ell^{-} \ell^{\prime-} \ell^{\prime+}$ decays can be found in "Appendix C".

${ }^{8}$ Our fits for the $H_{a}$ function are taken considering an interval for the
neutrino mass varying from $10^{-15}$ to $10 \mathrm{GeV}$.
9 These numbers correspond to the normal hierarchy $\left(m_{1}<m_{2}<m_{3}\right)$;
different (though very similar) values are reported for the inverted hierarchy $\left(m_{3}<m_{1}<m_{2}\right)$. Changing hierarchy is immaterial for our
Table 1 Values used for the evaluation of the branching fractions. We also assume a value of $m_{1}^{2}=(0.06)^{2} \mathrm{eV}^{2}$

\begin{tabular}{ll}
\hline Parameter & Value \\
\hline $\sin _{12}^{2}$ & $0.307(13)$ \\
$\sin _{23}^{2}$ & $0.51(4)$ \\
$\sin _{13}^{2}$ & $0.0210(11)$ \\
$\Delta m_{32}^{2}$ & $2.45(5) \times 10^{-3} \mathrm{eV}^{2}$ \\
$\Delta m_{21}^{2}$ & $7.53(18) \times 10^{-5} \mathrm{eV}^{2}$ \\
\hline
\end{tabular}

Table 2 Branching ratios for the $L^{-} \rightarrow \ell^{-} \ell^{\prime} \ell^{\prime+}$ decays (neglecting the penguin contributions), which are obtained using the current knowledge of the PMNS matrix. Our results are obtained taking into account only the contribution from the dominant $H_{a}$ function. The last column values correspond to the approximation where external masses and momenta are neglected [15]. Our results are smaller than those by three orders of magnitude, approximately

\begin{tabular}{lll}
\hline Decay channel & Our result & Ref. [15] \\
\hline$\mu^{-} \rightarrow e^{-} e^{+} e^{-}$ & $2.1 \times 10^{-56}$ & $2.6 \times 10^{-53}$ \\
$\tau^{-} \rightarrow e^{-} e^{+} e^{-}$ & $3.6 \times 10^{-57}$ & $4.5 \times 10^{-54}$ \\
$\tau^{-} \rightarrow \mu^{-} \mu^{+} \mu^{-}$ & $7.6 \times 10^{-56}$ & $9.7 \times 10^{-53}$ \\
$\tau^{-} \rightarrow e^{-} \mu^{+} \mu^{-}$ & $1.7 \times 10^{-57}$ & $2.2 \times 10^{-54}$ \\
$\tau^{-} \rightarrow \mu^{-} e^{+} e^{-}$ & $4.0 \times 10^{-56}$ & $5.0 \times 10^{-53}$ \\
\hline
\end{tabular}

Table 3 Branching ratios including all contributions (interferences are not neglected), which are obtained using the current knowledge of the PMNS matrix. Our results are obtained taking into account only the contribution from the dominant $H_{a}$ function. The last column values correspond to the approximation where external masses and momenta are neglected [15]. Our results are smaller than those by around one (two) orders of magnitude for the $\mu(\tau)$ decays

\begin{tabular}{lll}
\hline Decay channel & Our result & Ref. [15] \\
\hline$\mu^{-} \rightarrow e^{-} e^{+} e^{-}$ & $7.4 \times 10^{-55}$ & $8.5 \times 10^{-54}$ \\
$\tau^{-} \rightarrow e^{-} e^{+} e^{-}$ & $3.2 \times 10^{-56}$ & $1.4 \times 10^{-54}$ \\
$\tau^{-} \rightarrow \mu^{-} \mu^{+} \mu^{-}$ & $6.4 \times 10^{-55}$ & $3.2 \times 10^{-53}$ \\
$\tau^{-} \rightarrow e^{-} \mu^{+} \mu^{-}$ & $2.1 \times 10^{-56}$ & $9.4 \times 10^{-55}$ \\
$\tau^{-} \rightarrow \mu^{-} e^{+} e^{-}$ & $5.2 \times 10^{-55}$ & $2.1 \times 10^{-53}$ \\
\hline
\end{tabular}

We will first make a partial evaluation neglecting the penguin contributions (only box diagrams are considered), which yields the values in Table 2 .

Our final results, where the dominant penguin and box contributions are considered, are collected in Table 3, where they are compared to those obtained using Petcov's results [15] with updated input. Our predictions are even smaller than Petcov's updated results, as a consequence of keeping

Footnote 9 continued

numerical evaluations. We have verified that results are not sensitive to the lightest neutrino mass value, but only to the mass squared differences. 
external masses and momenta in our computations (Tables $4,5,6,7,8)$.

These extremely suppressed branching ratios for lepton flavor violating $L^{-} \rightarrow \ell^{-} \ell^{\prime-} \ell^{\prime+}$ decays due to massive light neutrinos are found at similar rates in the case of LFV Z [33] and Higgs boson decays [34].

\section{Conclusions}

We have revisited the $L^{-} \rightarrow \ell^{-} \ell^{\prime-} \ell^{\prime+}$ decays in the SM with massive neutrinos. We obtained expressions in terms of both Feynman parameters and scalar Passarino-Veltman functions for the relevant loop integrals of the (dominant) diagrams that involve two neutrino propagators considering non-vanishing masses and momenta of the external particles. Opposed to the previous calculation reported in Ref. [17], we found that all the different amplitudes for these processes are strongly suppressed (as they are proportional to the neutrino mass squared). In the particular case of the penguin contribution with two neutrino propagators, we highlight that it is crucial saving the dependence on the momentum transfer in the Feynman integrals in order to evaluate the amplitude in the physical region for the neutrino masses. This fact avoids the incorrect logarithmic divergent behavior in the amplitude claimed in Ref. [17]. As far as the box contribution is concerned, we found that the dominant term comes from $H_{a}$ function that is associated with a $(\mathrm{V}-\mathrm{A}) \times(\mathrm{V}-\mathrm{A})$ operator, and it is in good agreement with the approximation done in Ref. [15].

Current and forthcoming experiments were approaching the limits predicted by Ref. [17] on the SM prediction for the lepton flavor violating $\tau^{-} \rightarrow \mu^{-} \ell^{+} \ell^{-}(\ell=\mu, e)$ decays due to non-zero neutrino masses. This prediction was at odds with Ref. [15] corresponding computation for the $\mu^{-} \rightarrow e^{-} e^{+} e^{-}$decays predicting an extremely suppressed, unmeasurable branching ratio (as in $L^{-} \rightarrow \ell^{-} \gamma$ processes). The most important result of our analysis is the confirmation (in agreement with Ref. [15]) that any future observation of $L^{-} \rightarrow \ell^{-} \ell^{\prime-} \ell^{\prime+}$ decays would imply the existence of New Physics.

Acknowledgements The authors are indebted to Swagato Banerjee and Simon Eidelman for pointing us the interest of this calculation. We are thankful to Serguey Petcov for fruitful discussions. Finally, we also acknowledge support from Conacyt through projects FOINS-296-2016 (Fronteras de la Ciencia), and 236394 and 250628 (Ciencia Básica).

Data Availability Statement This manuscript has no associated data or the data will not be deposited. [Authors' comment: All data underlying the results are available as part of the article and no additional source data are required.]

Open Access This article is distributed under the terms of the Creative Commons Attribution 4.0 International License (http://creativecomm ons.org/licenses/by/4.0/), which permits unrestricted use, distribution,

and reproduction in any medium, provided you give appropriate credit to the original author(s) and the source, provide a link to the Creative Commons license, and indicate if changes were made. Funded by SCOAP ${ }^{3}$.

\section{A One-loop PaVe scalar functions}

In this appendix we collect the $\left\{\xi_{i_{j}}\right\}_{i=a, \ldots, f ; j=0, \ldots, 5}$ factors entering our results in Eq. (22):

$$
\begin{aligned}
& \xi_{a_{0}}=D_{F_{a}}, \\
& \xi_{a_{1}}=-m^{2}\left(m_{j}^{2}-m_{W}^{2}+M^{2}+q^{2}\right)+\left(M^{2}-q^{2}\right) \\
& \times\left(m_{j}^{2}-m_{W}^{2}+2 M^{2}-2 q^{2}\right)-m^{4}, \\
& \xi_{a_{2}}=-q^{2}\left(m_{j}^{2}+4 m^{2}-m_{W}^{2}+M^{2}\right)+\left(m^{2}-M^{2}\right) \\
& \times\left(m_{j}^{2}+2 m^{2}-m_{W}^{2}+M^{2}\right)+2 q^{4}, \\
& \xi_{a_{3}}=q^{2}\left(2 m_{j}^{2}+3 m^{2}-2 m_{W}^{2}+3 M^{2}-3 q^{2}\right), \\
& \xi_{a_{4}}=0 \text {, } \\
& \xi_{a_{5}}=-2 q^{2}\left(m^{2}\left(2 m_{j}^{2}-2 m_{W}^{2}+M^{2}-2 q^{2}\right)\right. \\
& \left.+\left(m_{j}^{2}-m_{W}^{2}+M^{2}-q^{2}\right)^{2}+m^{4}\right) . \\
& \xi_{b_{0}}=\xi_{b_{4}}=0 \text {, } \\
& \xi_{b_{1}}=-m M\left(m^{2}-M^{2}+q^{2}\right) \text {, } \\
& \xi_{b_{2}}=m M\left(m^{2}-M^{2}-q^{2}\right) \text {, } \\
& \xi_{b_{3}}=2 m M q^{2} \text {, } \\
& \xi_{b_{5}}=-m M q^{2}\left(2 m_{j}^{2}+m^{2}-2 m_{W}^{2}+M^{2}-q^{2}\right) \text {. } \\
& \xi_{c_{0}}=M^{2}\left(-m^{6}+m^{4}\left(3 M^{2}+q^{2}\right)+m^{2}\left(-3 M^{4}\right.\right. \\
& \left.\left.+2 M^{2} q^{2}+q^{4}\right)+\left(M^{2}-q^{2}\right)^{3}\right), \\
& \xi_{c_{1}}=M^{2}\left(-m^{4}\left(m_{j}^{2}-m_{W}^{2}+4 M^{2}+6 q^{2}\right)\right. \\
& +m^{2}\left(2 M^{2}\left(m_{j}^{2}-m_{W}^{2}-4 q^{2}\right)\right. \\
& \left.+q^{2}\left(-10 m_{j}^{2}+10 m_{W}^{2}+3 q^{2}\right)+5 M^{4}\right) \\
& \left.-\left(M^{2}-q^{2}\right)^{2}\left(m_{j}^{2}-m_{W}^{2}+2 M^{2}-2 q^{2}\right)+m^{6}\right), \\
& \xi_{c_{2}}=-q^{4}\left(m^{2}\left(3 m_{j}^{2}-3 m_{W}^{2}+7 M^{2}\right)\right. \\
& \left.+2 M^{2}\left(3 m_{j}^{2}-3 m_{W}^{2}+2 M^{2}\right)\right) \\
& -\left(m^{2}-2 M^{2}\right)\left(m^{2}-M^{2}\right)^{2}\left(m_{j}^{2}-m_{W}^{2}+M^{2}\right) \\
& +q^{2}\left(m^{4}\left(3 m_{j}^{2}-3 m_{W}^{2}+5 M^{2}\right)\right. \\
& +2 m^{2} M^{2}\left(m_{j}^{2}-m_{W}^{2}+2 M^{2}\right) \\
& \left.-M^{4}\left(-3 m_{j}^{2}+3 m_{W}^{2}+M^{2}\right)\right)+q^{6}\left(m_{j}^{2}-m_{W}^{2}+3 M^{2}\right), \\
& \xi_{c_{3}}=M^{2} q^{2}\left(m^{2}\left(6 m_{j}^{2}-6 m_{W}^{2}+4 M^{2}+4 q^{2}\right)\right. \\
& \left.-\left(M^{2}-q^{2}\right)\left(6 m_{j}^{2}-6 m_{W}^{2}+5 M^{2}-5 q^{2}\right)+m^{4}\right), \\
& \xi_{c_{4}}=\left(m_{j}^{2}-m_{W}^{2}\right)\left((m-M)^{2}-q^{2}\right) \\
& \times\left(m^{2}-M^{2}-q^{2}\right)\left((m+M)^{2}-q^{2}\right), \\
& \xi_{c_{5}}=-2 M^{2}\left(m^{6} m_{j}^{2}+m^{4}\left(M^{2}\left(2 q^{2}-3 m_{j}^{2}\right)\right.\right. \\
& \left.+q^{2}\left(m_{j}^{2}-2 m_{W}^{2}+q^{2}\right)\right)+m^{2}\left(M^{4}\left(3 m_{j}^{2}-q^{2}\right)\right.
\end{aligned}
$$




$$
\begin{aligned}
& +q^{2}\left(q^{2}\left(m_{j}^{2}-2 m_{W}^{2}\right)\right. \\
& \left.\left.+3\left(m_{j}^{2}-m_{W}^{2}\right)^{2}-2 q^{4}\right)+M^{2} q^{2}\left(3 q^{2}-2 m_{W}^{2}\right)\right) \\
& -\left(M^{2}-q^{2}\right)\left(M^{4}\left(m_{j}^{2}+q^{2}\right)\right. \\
& +2 M^{2} q^{2}\left(m_{j}^{2}-2 m_{W}^{2}-q^{2}\right)+q^{2}\left(-3 q^{2} m_{j}^{2}\right. \\
& \left.\left.\left.+3\left(m_{j}^{2}-m_{W}^{2}\right)^{2}+4 q^{2} m_{W}^{2}+q^{4}\right)\right)\right) \text {. } \\
& \xi_{d_{0}}=m^{2}\left(m^{6}-3 m^{4}\left(M^{2}+q^{2}\right)\right. \\
& +m^{2}\left(3 M^{4}+2 M^{2} q^{2}+3 q^{4}\right) \\
& \left.-\left(M^{2}-q^{2}\right)^{2}\left(M^{2}+q^{2}\right)\right) \text {, } \\
& \xi_{d_{1}}=-m^{6}\left(-2 m_{j}^{2}+2 m_{W}^{2}+5 M^{2}+q^{2}\right) \\
& +m^{4}\left(M^{2}\left(-5 m_{j}^{2}+5 m_{W}^{2}+4 q^{2}\right)\right. \\
& \left.+q^{2}\left(3 m_{j}^{2}-3 m_{W}^{2}-4 q^{2}\right)+4 M^{4}\right) \\
& -m^{2}\left(M^{2}-q^{2}\right)\left(-4 M^{2}\left(m_{j}^{2}-m_{W}^{2}+q^{2}\right)\right. \\
& \left.+3 q^{2}\left(-2 m_{j}^{2}+2 m_{W}^{2}+q^{2}\right)+M^{4}\right) \\
& -\left(M^{2}-q^{2}\right)^{3}\left(m_{j}^{2}-m_{W}^{2}\right)+2 m^{8}, \\
& \xi_{d_{2}}=m^{2}\left(q^{4}\left(-m_{j}^{2}-6 m^{2}+m_{W}^{2}+3 M^{2}\right)\right. \\
& -\left(m^{2}-M^{2}\right)^{2}\left(m_{j}^{2}+2 m^{2}-m_{W}^{2}-M^{2}\right) \\
& +2 q^{2}\left(m^{2}\left(m_{j}^{2}-m_{W}^{2}-4 M^{2}\right)\right. \\
& \left.\left.+M^{2}\left(-5 m_{j}^{2}+5 m_{W}^{2}-3 M^{2}\right)+3 m^{4}\right)+2 q^{6}\right), \\
& \xi_{d_{3}}=m^{2} q^{2}\left(2 q^{2}\left(3 m_{j}^{2}+5 m^{2}-3 m_{W}^{2}+2 M^{2}\right)\right. \\
& \left.-\left(m^{2}-M^{2}\right)\left(6 m_{j}^{2}+5 m^{2}-6 m_{W}^{2}+M^{2}\right)-5 q^{4}\right), \\
& \xi_{d_{4}}=\left(m_{j}^{2}-m_{W}^{2}\right)\left(-\left((m-M)^{2}-q^{2}\right)\right) \\
& \times\left((m+M)^{2}-q^{2}\right)\left(m^{2}-M^{2}+q^{2}\right), \\
& \xi_{d_{5}}=2 m^{2}\left(m^{6}\left(m_{j}^{2}+q^{2}\right)+m^{4}\left(M^{2}\left(q^{2}-3 m_{j}^{2}\right)\right.\right. \\
& \left.+q^{2}\left(m_{j}^{2}-4 m_{W}^{2}-3 q^{2}\right)\right)+m^{2}\left(M^{4}\left(3 m_{j}^{2}-2 q^{2}\right)\right. \\
& +q^{2}\left(-5 q^{2} m_{j}^{2}+3\left(m_{j}^{2}-m_{W}^{2}\right)^{2}+8 q^{2} m_{W}^{2}+3 q^{4}\right) \\
& \left.+M^{2} q^{2}\left(2 m_{W}^{2}-3 q^{2}\right)\right)-M^{6} m_{j}^{2} \\
& -M^{4} q^{2}\left(m_{j}^{2}-2 m_{W}^{2}+q^{2}\right) \\
& +M^{2} q^{2}\left(-q^{2}\left(m_{j}^{2}-2 m_{W}^{2}\right)-3\left(m_{j}^{2}-m_{W}^{2}\right)^{2}+2 q^{4}\right) \\
& +q^{4}\left(3 m_{j}^{2}\left(2 m_{W}^{2}+q^{2}\right)\right. \\
& \left.\left.-3 m_{j}^{4}-\left(m_{W}^{2}+q^{2}\right)\left(3 m_{W}^{2}+q^{2}\right)\right)\right) \text {. } \\
& \xi_{e_{0}}=-M^{2}\left(3 m^{6}-m^{4}\left(5 M^{2}+7 q^{2}\right)+m^{2}\left(M^{4}\right.\right. \\
& \left.\left.-6 M^{2} q^{2}+5 q^{4}\right)+\left(M^{2}-q^{2}\right)^{3}\right), \\
& \xi_{e_{1}}=M^{2}\left(m^{4}\left(-11 m_{j}^{2}+11 m_{W}^{2}+2 q^{2}\right)\right. \\
& +m^{2}\left(2 M^{2}\left(5 m_{j}^{2}-5 m_{W}^{2}-4 q^{2}\right)\right. \\
& +q^{2}\left(-2 m_{j}^{2}+2 m_{W}^{2}+5 q^{2}\right) \\
& \left.+3 M^{4}\right)+\left(M^{2}-q^{2}\right)^{2}\left(m_{j}^{2}-m_{W}^{2}\right. \\
& \left.\left.+2 M^{2}-2 q^{2}\right)-5 m^{6}\right) \text {, } \\
& \xi_{e_{2}}=m^{6}\left(-m_{j}^{2}+m_{W}^{2}+3 M^{2}\right) \\
& +m^{4}\left(M^{2}\left(6 m_{j}^{2}-6 m_{W}^{2}-7 q^{2}\right)\right. \\
& \left.+3 q^{2}\left(m_{j}^{2}-m_{W}^{2}\right)+2 M^{4}\right) \\
& +m^{2}\left(M^{4}\left(3 m_{j}^{2}-3 m_{W}^{2}+4 q^{2}\right)\right. \\
& +M^{2} q^{2}\left(-2 m_{j}^{2}+2 m_{W}^{2}+5 q^{2}\right)
\end{aligned}
$$

$$
\begin{aligned}
& \left.+3 q^{4}\left(m_{W}^{2}-m_{j}^{2}\right)-M^{6}\right) \\
& -\left(M^{2}-q^{2}\right)\left(M^{4}\left(8 m_{j}^{2}-8 m_{W}^{2}-3 q^{2}\right)\right. \\
& \left.-M^{2} q^{2}\left(3 m_{j}^{2}-3 m_{W}^{2}+q^{2}\right)+q^{4}\left(m_{j}^{2}-m_{W}^{2}\right)+4 M^{6}\right), \\
& \xi_{e_{3}}=M^{2}\left(-2 q^{4}\left(m_{j}^{2}+5 m^{2}-m_{W}^{2}+2 M^{2}\right)\right. \\
& +q^{2}\left(5 m^{2}-M^{2}\right)\left(2 m_{j}^{2}+m^{2}-2 m_{W}^{2}+M^{2}\right) \\
& \left.+2\left(m^{2}-M^{2}\right)^{2}\left(2 m_{j}^{2}+m^{2}-2 m_{W}^{2}+M^{2}\right)+3 q^{6}\right), \\
& \xi_{e_{4}}=\left(m_{j}^{2}-m_{W}^{2}\right)\left((m-M)^{2}-q^{2}\right) \\
& \times\left(m^{2}+3 M^{2}-q^{2}\right)\left((m+M)^{2}-q^{2}\right), \\
& \xi_{e_{5}}=-2 M^{2}\left(m^{6} m_{j}^{2}+m^{4}\left(M^{2}\left(2 q^{2}-3 m_{j}^{2}\right)\right.\right. \\
& \left.+q^{2}\left(m_{j}^{2}-2 m_{W}^{2}+q^{2}\right)\right)+m^{2}\left(M^{4}\left(3 m_{j}^{2}-q^{2}\right)\right. \\
& +q^{2}\left(q^{2}\left(m_{j}^{2}-2 m_{W}^{2}\right)\right. \\
& \left.\left.+3\left(m_{j}^{2}-m_{W}^{2}\right)^{2}-2 q^{4}\right)+M^{2} q^{2}\left(3 q^{2}-2 m_{W}^{2}\right)\right) \\
& -\left(M^{2}-q^{2}\right)\left(M^{4}\left(m_{j}^{2}+q^{2}\right)\right. \\
& +2 M^{2} q^{2}\left(m_{j}^{2}-2 m_{W}^{2}-q^{2}\right) \\
& \left.\left.+q^{2}\left(-3 q^{2} m_{j}^{2}+3\left(m_{j}^{2}-m_{W}^{2}\right)^{2}+4 q^{2} m_{W}^{2}+q^{4}\right)\right)\right) \text {. } \\
& \xi_{f_{0}}=-m^{2}\left(-m^{6}-m^{4}\left(M^{2}-3 q^{2}\right)\right. \\
& +m^{2}\left(5 M^{4}+6 M^{2} q^{2}-3 q^{4}\right) \\
& \left.-\left(M^{2}-q^{2}\right)^{2}\left(3 M^{2}-q^{2}\right)\right), \\
& \xi_{f_{1}}=m^{6}\left(8 m_{j}^{2}-8 m_{W}^{2}+M^{2}-7 q^{2}\right) \\
& +m^{4}\left(M^{2}\left(-3 m_{j}^{2}+3 m_{W}^{2}-4 q^{2}\right)\right. \\
& \left.+q^{2}\left(-11 m_{j}^{2}+11 m_{W}^{2}+2 q^{2}\right)-2 M^{4}\right) \\
& -m^{2}\left(M^{2}-q^{2}\right)\left(M^{2}\left(6 m_{j}^{2}-6 m_{W}^{2}-4 q^{2}\right)\right. \\
& \left.+q^{2}\left(4 m_{j}^{2}-4 m_{W}^{2}+q^{2}\right)+3 M^{4}\right) \\
& +\left(M^{2}-q^{2}\right)^{3}\left(m_{j}^{2}-m_{W}^{2}\right)+4 m^{8}, \\
& \xi_{f_{2}}=m^{2}\left(m^{4}\left(-m_{j}^{2}+m_{W}^{2}-3 M^{2}+6 q^{2}\right)\right. \\
& +2 m^{2}\left(M^{2}\left(-5 m_{j}^{2}+5 m_{W}^{2}+4 q^{2}\right)\right. \\
& \left.+q^{2}\left(m_{j}^{2}-m_{W}^{2}-3 q^{2}\right)\right) \\
& +M^{4}\left(11 m_{j}^{2}-11 m_{W}^{2}-2 q^{2}\right) \\
& +M^{2} q^{2}\left(2 m_{j}^{2}-2 m_{W}^{2}-5 q^{2}\right) \\
& \left.+q^{4}\left(-m_{j}^{2}+m_{W}^{2}+2 q^{2}\right)-2 m^{6}+5 M^{6}\right), \\
& \xi_{f_{3}}=m^{2}\left(2 q^{4}\left(m_{j}^{2}+2 m^{2}-m_{W}^{2}+5 M^{2}\right)\right. \\
& +q^{2}\left(m^{2}-5 M^{2}\right)\left(2 m_{j}^{2}+m^{2}-2 m_{W}^{2}+M^{2}\right) \\
& \left.-2\left(m^{2}-M^{2}\right)^{2}\left(2 m_{j}^{2}+m^{2}-2 m_{W}^{2}+M^{2}\right)-3 q^{6}\right), \\
& \xi_{f_{4}}=\left(m_{j}^{2}-m_{W}^{2}\right)\left(-\left((m-M)^{2}-q^{2}\right)\right) \\
& \times\left(3 m^{2}+M^{2}-q^{2}\right)\left((m+M)^{2}-q^{2}\right), \\
& \xi_{f_{5}}=2 m^{2}\left(q^{6}\left(m_{j}^{2}+3 m^{2}-2 m_{W}^{2}+4 M^{2}\right)\right. \\
& +(m-M)^{2}(m+M)^{2}\left(m^{2}\left(3 m_{j}^{2}-2 m_{W}^{2}+2 M^{2}\right)\right. \\
& +M^{2}\left(5 m_{j}^{2}-2 m_{W}^{2}\right) \\
& \left.+2\left(m_{j}^{2}-m_{W}^{2}\right)^{2}\right)-q^{4}\left(-2 m_{W}^{2}\left(m_{j}^{2}+m^{2}+4 M^{2}\right)\right. \\
& \left.-m^{2} m_{j}^{2}+3 M^{2} m_{j}^{2}+m_{j}^{4}+3 m^{4}+5 m^{2} M^{2}+m_{W}^{4}+5 M^{4}\right) \\
& +q^{2}\left(-m^{4}\left(5 m_{j}^{2}-2 m_{W}^{2}+M^{2}\right)\right. \\
& +m^{2}\left(-\left(m_{j}^{2}-m_{W}^{2}\right)^{2}-6 M^{2} m_{W}^{2}+2 M^{4}\right)
\end{aligned}
$$




$$
\begin{aligned}
& +M^{2}\left(-M^{2}\left(3 m_{j}^{2}+4 m_{W}^{2}\right)\right. \\
& \left.\left.\left.+5\left(m_{j}^{2}-m_{W}^{2}\right)^{2}+2 M^{4}\right)+m^{6}\right)-q^{8}\right) .
\end{aligned}
$$

As far as the $\chi_{k}$ factors entering the $H_{P V_{a}}$ functions in Eq. (53), they are given as follows

$$
\begin{aligned}
& \chi_{k_{1}}=m^{2}\left(s _ { 1 2 } \left(m_{i}^{2}+2 m_{j}^{2}-3 m_{\ell^{\prime}}^{2}-3 m_{W}^{2}\right.\right. \\
& \left.\left.+2 s_{12}+s_{13}\right)+2 M^{2}\left(m_{j}^{2}-m_{\ell^{\prime}}^{2}-m_{W}^{2}\right)\right) \\
& +M^{2} s_{12}\left(m_{i}^{2}+2 m_{j}^{2}-3 m_{\ell^{\prime}}^{2}-3 m_{W}^{2}+2 s_{12}\right. \\
& \left.+s_{13}\right)-s_{12}\left(m_{i}^{2}\left(-2 m_{\ell^{\prime}}^{2}+s_{12}+2 s_{13}\right)\right. \\
& +s_{12} m_{j}^{2}+2 m_{\ell^{\prime}}^{2}\left(m_{W}^{2}-s_{12}\right) \\
& \left.-\left(s_{12}+s_{13}\right)\left(2 m_{W}^{2}-s_{12}\right)\right) \\
& +m^{4}\left(-m_{j}^{2}+m_{\ell^{\prime}}^{2}+m_{W}^{2}-s_{12}\right) \\
& +M^{4}\left(-m_{j}^{2}+m_{\ell^{\prime}}^{2}+m_{W}^{2}-s_{12}\right), \\
& \chi_{k_{2}}=-s_{12}\left(-4 m_{i}^{2} m_{\ell^{\prime}}^{2}+s_{12} m_{i}^{2}\right. \\
& -m^{2}\left(m_{j}^{2}-3 m_{\ell^{\prime}}^{2}-m_{W}^{2}+s_{12}\right)-M^{2} \\
& \times\left(m_{j}^{2}-3 m_{\ell^{\prime}}^{2}-m_{W}^{2}+s_{12}\right)-2 m_{j}^{2} m_{\ell^{\prime}}^{2} \\
& +s_{12} m_{j}^{2}+2 s_{13} m_{j}^{2}-4 s_{12} m_{\ell^{\prime}}^{2}-2 s_{13} m_{\ell^{\prime}}^{2} \\
& +6 m_{\ell^{\prime}}^{2} m_{W}^{2}+2 m_{\ell^{\prime}}^{4}-2 s_{12} m_{W}^{2}-2 s_{13} m_{W}^{2} \\
& \left.+s_{12}^{2}+s_{12} s_{13}\right) \text {, } \\
& \chi_{k_{3}}=-m^{2}\left(m_{i}^{2}\left(s_{12}-2 m_{\ell^{\prime}}^{2}\right)\right. \\
& +M^{2}\left(m_{j}^{2}+m_{\ell^{\prime}}^{2}-m_{W}^{2}-s_{12}\right) \\
& +s_{13}\left(m_{j}^{2}-m_{\ell^{\prime}}^{2}-m_{W}^{2}+2 s_{12}\right) \\
& -m_{j}^{2} m_{\ell^{\prime}}^{2}+2 s_{12} m_{j}^{2}-4 s_{12} m_{\ell^{\prime}}^{2}+3 m_{\ell^{\prime}}^{2} m_{W}^{2} \\
& \left.+m_{\ell^{\prime}}^{4}-3 s_{12} m_{W}^{2}+2 s_{12}^{2}\right)-M^{2}\left(2 m_{i}^{2} m_{\ell^{\prime}}^{2}\right. \\
& +m_{j}^{2}\left(m_{\ell^{\prime}}^{2}+s_{12}-s_{13}\right) \\
& +s_{13}\left(m_{\ell^{\prime}}^{2}+m_{W}^{2}+s_{12}\right)-3 m_{\ell^{\prime}}^{2} m_{W}^{2}-m_{\ell^{\prime}}^{4} \\
& \left.-s_{12} m_{W}^{2}+s_{12}^{2}\right)+s_{12}\left(m_{i}^{2}\left(-3 m_{\ell^{\prime}}^{2}+s_{12}+s_{13}\right)\right. \\
& +m_{j}^{2}\left(-m_{\ell^{\prime}}^{2}+s_{12}+s_{13}\right)+\left(2 m_{\ell^{\prime}}^{2}-s_{12}-s_{13}\right) \\
& \left.\times\left(m_{\ell^{\prime}}^{2}+2 m_{W}^{2}-s_{12}-s_{13}\right)\right) \\
& +m^{4}\left(m_{j}^{2}-m_{\ell^{\prime}}^{2}-m_{W}^{2}+s_{12}\right)+2 M^{4} m_{\ell^{\prime}}^{2}, \\
& \chi_{k_{4}}=-m^{2}\left(2 m_{i}^{2} m_{\ell^{\prime}}^{2}+M^{2}\left(m_{j}^{2}+m_{\ell^{\prime}}^{2}-m_{W}^{2}-s_{12}\right)\right. \\
& +s_{13}\left(-m_{j}^{2}+m_{\ell^{\prime}}^{2}+m_{W}^{2}+s_{12}\right)+m_{j}^{2} m_{\ell^{\prime}}^{2} \\
& \left.+s_{12} m_{j}^{2}-3 m_{\ell^{\prime}}^{2} m_{W}^{2}-m_{\ell^{\prime}}^{4}-s_{12} m_{W}^{2}+s_{12}^{2}\right) \\
& +M^{2}\left(m_{i}^{2}\left(2 m_{\ell^{\prime}}^{2}-s_{12}\right)+m_{j}^{2}\left(m_{\ell^{\prime}}^{2}-2 s_{12}-s_{13}\right)\right. \\
& +s_{13}\left(m_{\ell^{\prime}}^{2}+m_{W}^{2}-2 s_{12}\right)+4 s_{12} m_{\ell^{\prime}}^{2}-3 m_{\ell^{\prime}}^{2} m_{W}^{2} \\
& \left.-m_{\ell^{\prime}}^{4}+3 s_{12} m_{W}^{2}-2 s_{12}^{2}\right)+s_{12}\left(m _ { i } ^ { 2 } \left(-3 m_{\ell^{\prime}}^{2}\right.\right. \\
& \left.+s_{12}+s_{13}\right) \\
& +m_{j}^{2}\left(-m_{\ell^{\prime}}^{2}+s_{12}+s_{13}\right)+\left(2 m_{\ell^{\prime}}^{2}-s_{12}-s_{13}\right) \\
& \left.\times\left(m_{\ell^{\prime}}^{2}+2 m_{W}^{2}-s_{12}-s_{13}\right)\right) \\
& +M^{4}\left(m_{j}^{2}-m_{\ell^{\prime}}^{2}-m_{W}^{2}+s_{12}\right)+2 m^{4} m_{\ell^{\prime}}^{2}, \\
& \chi_{k_{5}}=2 m^{2}\left(s _ { 1 2 } \left(m_{i}^{2}\left(m_{j}^{2}-3 m_{\ell^{\prime}}^{2}-m_{W}^{2}+s_{12}\right)\right.\right. \\
& +m_{j}^{2}\left(-3 m_{\ell^{\prime}}^{2}-3 m_{W}^{2}+2 s_{12}+s_{13}\right) \\
& +m_{j}^{4}-3 s_{12} m_{\ell^{\prime}}^{2}-s_{13} m_{\ell^{\prime}}^{2} \\
& +4 m_{\ell^{\prime}}^{2} m_{W}^{2}+2 m_{\ell^{\prime}}^{4}-3 s_{12} m_{W}^{2}-3 s_{13} m_{W}^{2}
\end{aligned}
$$

$$
\begin{aligned}
& \left.+q^{2}(x-1)\right)+\frac{\left(\theta_{m}-\theta_{M}\right)}{2 q^{2}}, \\
\int_{0}^{1-x} \frac{y^{2} d y}{D_{j}}= & \frac{\left(T_{-}-T_{+}\right)}{\Lambda q^{4}}\left(2 q^{2}(x-1) m_{j}^{2}\right. \\
& +x^{2}\left(m^{2}-M^{2}\right)^{2}-2 q^{2} x\left(m^{2}(x-1)+m_{W}^{2}\right) \\
& \left.+q^{4}(x-1)^{2}\right) \\
& -\frac{\left(\theta_{m}-\theta_{M}\right)}{2 q^{4}}\left(x\left(M^{2}-m^{2}\right)+q^{2}(x-1)\right)+\frac{1-x}{q^{2}}, \\
\int_{0}^{1-x} \ln \left(D_{j}\right) d y= & \frac{\Lambda\left(T_{-}-T_{+}\right)}{q^{2}} \\
& +\frac{\left(\theta_{m}-\theta_{M}\right)\left(x\left(M^{2}-m^{2}\right)+q^{2}(x-1)\right)}{2 q^{2}} \\
& -(x-1)\left(\operatorname { l o g } \left(x\left(m^{2}(x-1)+m_{W}^{2}\right)\right.\right. \\
& \left.\left.-(x-1) m_{j}^{2}\right)-2\right),
\end{aligned}
$$$$
\int_{0}^{1-x} \frac{y d y}{D_{j}}=\frac{\left(T_{+}-T_{-}\right)}{q^{2} \Lambda}\left(x\left(M^{2}-m^{2}\right)\right.
$$

where we have defined the functions 
ond terms in Eqs. (102) and (103), respectively. Therefore, $\left|\mathscr{M}^{2}\right|$ is given by

$$
\begin{aligned}
\Lambda & =\sqrt{-4 q^{2}(x-1) m_{j}^{2}+2 q^{2} x\left(m^{2}(x-1)+2 m_{W}^{2}+M^{2}(x-1)\right)-x^{2}\left(m^{2}-M^{2}\right)^{2}+q^{4}\left(-(x-1)^{2}\right)} \\
T_{+} & =\tan ^{-1}\left(\frac{x\left(M^{2}-m^{2}\right)+q^{2}(x-1)}{\Lambda}\right) \\
T_{-} & =\tan ^{-1}\left(\frac{x\left(M^{2}-m^{2}\right)-q^{2}(x-1)}{\Lambda}\right) \\
\theta_{M} & =\log \left((x-1) m_{j}^{2}-x\left(m_{W}^{2}+M^{2}(x-1)\right)\right) \\
\theta_{m} & =\log \left((x-1) m_{j}^{2}-x\left(m_{W}^{2}+m^{2}(x-1)\right)\right)
\end{aligned}
$$

\section{Kinematics for the $L^{-}(P, M) \rightarrow \ell^{-}(p, m)$ $\ell^{\prime-}\left(p_{1}, m_{\ell^{\prime}}\right) \ell^{\prime+}\left(p_{2}, m_{\ell^{\prime}}\right)$ decays}

Because of the necessity of anti-symmetrizing the amplitude when $\ell=\ell^{\prime}$, the total contribution for the sum of the penguin and box diagrams, in this case, is given by

$$
\begin{aligned}
\mathscr{M}_{\ell=\ell^{\prime}}= & i G_{F}^{2} m_{W}^{2}\left(-\frac{\beta_{F_{a}}}{4}+8 m_{W}^{2} \beta_{H_{a}}\right) \bar{u}(p) \gamma_{\lambda}\left(1-\gamma_{5}\right) u(P) \\
& \times \bar{u}\left(p_{1}\right) \gamma^{\lambda}\left(1-\gamma_{5}\right) v\left(p_{2}\right)-\left(p \leftrightarrow p_{1}\right) \\
& +i G_{F}^{2} m_{W}^{2} s_{W}^{2} \beta_{F_{a}} \bar{u}(p) \gamma_{\lambda}\left(1-\gamma_{5}\right) u(P) \\
& \times \bar{u}\left(p_{1}\right) \gamma^{\lambda} v\left(p_{2}\right)-\left(p \leftrightarrow p_{1}\right) .
\end{aligned}
$$

On the other hand, when $\ell \neq \ell^{\prime}$, there is only one penguin diagram since the vertex of a neutral $Z$ boson with a pair of fermions is flavor-conserving. Besides, we have to add the box diagram interchanging $\ell^{-}(p) \leftrightarrow \ell^{\prime-}\left(p_{1}\right)$. Therefore, we have

$$
\begin{aligned}
\mathscr{M}_{\ell \neq \ell^{\prime}}= & i G_{F}^{2} m_{W}^{2}\left(-\frac{\beta_{F_{a}}}{4}+8 m_{W}^{2} \beta_{H_{a}}\right) \\
& \times \bar{u}(p) \gamma_{\lambda}\left(1-\gamma_{5}\right) u(P) \\
& \times \bar{u}\left(p_{1}\right) \gamma^{\lambda}\left(1-\gamma_{5}\right) v\left(p_{2}\right) \\
& +i G_{F}^{2} m_{W}^{2} s_{W}^{2} \beta_{F_{a}} \bar{u}(p) \gamma_{\lambda}\left(1-\gamma_{5}\right) u(P) \times \bar{u}\left(p_{1}\right) \gamma^{\lambda} v\left(p_{2}\right) \\
& +8 i G_{F}^{2} m_{W}^{4} \hat{\beta}_{H_{a}} \bar{u}\left(p_{1}\right) \gamma_{\lambda}\left(1-\gamma_{5}\right) u(P) \\
& \times \bar{u}(p) \gamma^{\lambda}\left(1-\gamma_{5}\right) v\left(p_{2}\right),
\end{aligned}
$$

where $\beta_{H_{a}}$ has been defined in the main text and

$$
\hat{\beta}_{H_{a}}=\sum_{j, i} U_{L j} U_{\ell i}^{*} U_{\ell^{\prime} j} U_{\ell^{\prime} i}^{*} H_{a}\left(m_{i}^{2}, m_{j}^{2}\right) .
$$

In Petcov's approximation, taking only the dominant term and since the contribution of the penguin and box diagrams have opposite sign, the dominant terms are given by the sec-
$\left|\mathscr{M}^{2}\right|=\frac{G_{F}^{4} s_{W}^{4}}{4 \pi^{4}}\left(\sum_{j} U_{L j} U_{l j}^{*} m_{j}^{2} \log \left(\frac{m_{W}^{2}}{m_{j}^{2}}\right)\right)^{2} T_{s}$,

where

$$
\begin{aligned}
T_{s}= & -16\left(-4 s_{13} m_{\ell^{\prime}}^{2}+2 m_{\ell^{\prime}}^{4}-s_{12}\left(m^{2}+M^{2}-2 s_{13}\right)\right. \\
& \left.+2\left(m^{2}-s_{13}\right)\left(M^{2}-s_{13}\right)+s_{12}^{2}\right)
\end{aligned}
$$

for $\ell \neq \ell^{\prime}$, and

$$
\begin{aligned}
T_{s}= & -16\left(-s_{13}\left(5 m^{2}+M^{2}\right)+s_{13}^{2}\right. \\
& +2\left(9 m^{4}-2 s_{13}\left(4 m^{2}+M^{2}\right)\right. \\
& +s_{12}\left(-3 m^{2}-M^{2}+s_{13}\right)+5 m^{2} M^{2} \\
& \left.\left.+s_{12}^{2}+2 s_{13}^{2}\right)\right)
\end{aligned}
$$

when $\ell=\ell^{\prime}\left(m=m_{\ell^{\prime}}\right)$.

The unpolarized differential decay width for the $L^{-}(P) \rightarrow$ $\ell^{-}(p) \ell^{\prime-}\left(p_{1}\right) \ell^{\prime+}\left(p_{2}\right)$ decays is given by

$$
\Gamma=\frac{1 / N}{4(4 \pi)^{3} M^{3}} \int|\mathscr{M}|^{2} d s_{12} d s_{13},
$$

where $N$ is the number of identical particles in the final state and $s_{12}=\left(p_{1}+p_{2}\right)^{2}=q^{2}$ and $s_{13}=\left(p_{1}+p\right)^{2}$. The corresponding integration limits are given by

$$
\begin{aligned}
s_{13}^{ \pm}= & \frac{1}{2}\left(M^{2}+m^{2}-s_{12}\right)+m_{\ell^{\prime}}^{2} \\
& \pm \frac{\sqrt{\lambda\left(M^{2}, s_{12}, m^{2}\right) \lambda\left(s_{12}, m_{\ell^{\prime}}^{2}, m_{\ell^{\prime}}^{2}\right)}}{2 s_{12}},
\end{aligned}
$$

and

$4 m_{\ell^{\prime}}^{2} \leq s_{12} \leq(M-m)^{2}$. 


\section{$D$ Fits for $Z \tau \mu, Z \tau e$ and $Z \mu e$ effective vertices}

The numerical values for the $Q_{k}$ and $R_{k}$ factors involved in of our fits for the $Z \tau \mu, Z \tau e$ and $Z \mu e$ effective vertices are given as follows (see Tables 4, 5, 6, 7, 8):

Table 4 Values for the $Q_{R_{k}}\left(Q_{I_{k}}\right)$ and $R_{R_{k}}\left(R_{I_{k}}\right)$ coefficients of the $Z \tau \mu$ vertex for $q^{2}=4 m_{\mu}^{2}$

\begin{tabular}{lllll}
\hline$Z \tau \mu\left(q^{2}=4 m_{\mu}^{2}\right)$ & $Q_{R_{k}}$ & $R_{R_{k}}$ & $Q_{I_{k}}$ & $R_{I_{k}}$ \\
\hline$a$ & 4.63706 & 11.5451 & $-7.14896 \times 10^{-6}$ & 3.4098 \\
$b$ & $1.38093 \times 10^{-5}$ & $-3.31777 \times 10^{-4}$ & $9.85094 \times 10^{-11}$ & $-6.76208 \times 10^{-5}$ \\
$c$ & $-1.49047 \times 10^{-5}$ & $3.62348 \times 10^{-3}$ & $-7.884 \times 10^{-10}$ & $5.4035 \times 10^{-4}$ \\
$d$ & $-9.20638 \times 10^{-6}$ & $1.2469 \times 10^{-4}$ & $-4.9267 \times 10^{-11}$ & $3.38191 \times 10^{-5}$ \\
$e$ & $2.04592 \times 10^{-3}$ & 191.959 & $4.69628 \times 10^{-4}$ & -126.096 \\
$f$ & $-1.26365 \times 10^{-5}$ & -11.8554 & $-2.95163 \times 10^{-5}$ & 8.05527 \\
\hline
\end{tabular}

Table 5 Same as Table 4 but considering $q^{2}=4 m_{e}^{2}$

\begin{tabular}{lllll}
\hline$Z \tau \mu\left(q^{2}=4 m_{e}^{2}\right)$ & $Q_{R_{k}}$ & $R_{R_{k}}$ & $Q_{I_{k}}$ & $R_{I_{k}}$ \\
\hline$a$ & 4.63709 & 22.2936 & $-1.6966 \times 10^{-10}$ & 3.40516 \\
$b$ & $1.3809 \times 10^{-5}$ & $-6.24913 \times 10^{-4}$ & $2.31697 \times 10^{-15}$ & $-6.71321 \times 10^{-5}$ \\
$c$ & $-1.49044 \times 10^{-4}$ & $-3.92512 \times 10^{-2}$ & $-1.89825 \times 10^{-14}$ & $6.2734 \times 10^{-4}$ \\
$d$ & $-9.20617 \times 10^{-6}$ & -0.191951 & $-1.0909 \times 10^{-15}$ & $2.6232 \times 10^{-5}$ \\
$e$ & $3.63186 \times 10^{-3}$ & $8.17424 \times 10^{6}$ & $4.74754 \times 10^{-4}$ & $-5.3963 \times 10^{6}$ \\
$f$ & $-2.2432 \times 10^{-4}$ & $-504,881$ & $-2.93231 \times 10^{-5}$ & 333,301 \\
\hline
\end{tabular}

Table 6 Values for the $Q_{R_{k}}\left(Q_{I_{k}}\right)$ and $R_{R_{k}}\left(R_{I_{k}}\right)$ coefficients of the $Z \tau e$ vertex for $q^{2}=4 m_{\mu}^{2}$

\begin{tabular}{lllll}
\hline$Z \tau e\left(q^{2}=4 m_{\mu}^{2}\right)$ & $Q_{R_{k}}$ & $R_{R_{k}}$ & $Q_{I_{k}}$ & $R_{I_{k}}$ \\
\hline$a$ & 4.63706 & 11.5451 & $-7.14896 \times 10^{-6}$ & 3.4098 \\
$b$ & $6.72054 \times 10^{-8}$ & $-1.61465 \times 10^{-6}$ & $4.79412 \times 10^{-13}$ & $-3.29087 \times 10^{-7}$ \\
$c$ & $-1.49047 \times 10^{-4}$ & $3.61659 \times 10^{-3}$ & $-7.88464 \times 10^{-10}$ & $5.4042 \times 10^{-4}$ \\
$d$ & $-3.86832 \times 10^{-8}$ & $-5.66645 \times 10^{-3}$ & $-2.39753 \times 10^{-13}$ & $1.64583 \times 10^{-7}$ \\
$e$ & $2.04592 \times 10^{-3}$ & 191.962 & $4.69628 \times 10^{-4}$ & -126.095 \\
$f$ & $-6.09267 \times 10^{-7}$ & $-5.92939 \times 10^{-2}$ & $-1.43646 \times 10^{-7}$ & $3.920023 \times 10^{-2}$ \\
\hline
\end{tabular}

Table 7 Same as Table 6 but considering $q^{2}=4 m_{e}^{2}$

\begin{tabular}{lllll}
\hline$Z \tau e\left(q^{2}=4 m_{e}^{2}\right)$ & $Q_{R_{k}}$ & $R_{R_{k}}$ & $Q_{I_{k}}$ & $R_{I_{k}}$ \\
\hline$a$ & 4.63709 & 22.2262 & $-1.6966 \times 10^{-10}$ & 3.40516 \\
$b$ & $6.72036 \times 10^{-8}$ & $-3.05754 \times 10^{-4}$ & $1.12759 \times 10^{-17}$ & $-3.26709 \times 10^{-7}$ \\
$c$ & $-1.49043 \times 10^{-4}$ & -.107576 & $-1.89741 \times 10^{-14}$ & $6.26186 \times 10^{-4}$ \\
$d$ & $-4.95278 \times 10^{-8}$ & 19.097 & $-5.68205 \times 10^{-18}$ & $1.64383 \times 10^{-7}$ \\
$e$ & $3.63189 \times 10^{-3}$ & $8.17296 \times 10^{6}$ & $4.74754 \times 10^{-4}$ & $-5.3963 \times 10^{6}$ \\
$f$ & $-1.08821 \times 10^{-6}$ & -2468.32 & $-1.42705 \times 10^{-7}$ & 1622.06 \\
\hline
\end{tabular}


Table 8 Values for the $Q_{R_{k}}\left(Q_{I_{k}}\right)$ and $R_{R_{k}}\left(R_{I_{k}}\right)$ coefficients of the $Z \mu e$ vertex for $q^{2}=4 m_{\mu}^{2}$

\begin{tabular}{lllll}
\hline$Z \mu e\left(q^{2}=4 m_{e}^{2}\right)$ & $Q_{R_{k}}$ & $R_{R_{k}}$ & $Q_{I_{k}}$ & $R_{I_{k}}$ \\
\hline$a$ & 4.63701 & 31.6578 & $-1.55723 \times 10^{-10}$ & 1.15008 \\
$b$ & $4.15019 \times 10^{-9}$ & $-1.01036 \times 10^{-7}$ & $7.02341 \times 10^{-19}$ & $-2.03165 \times 10^{-8}$ \\
$c$ & $-5.6794 \times 10^{-7}$ & -2.40973 & $-1.60743 \times 10^{-13}$ & $2.42044 \times 10^{-3}$ \\
$d$ & $-9.81338 \times 10^{-8}$ & 2359.07 & $-1.54223 \times 10^{-16}$ & $-2.54211 \times 10^{-6}$ \\
$e$ & $1.37244 \times 10^{-5}$ & 32441.2 & $1.78855 \times 10^{-6}$ & -20427.4 \\
$f$ & $-8.46878 \times 10^{-8}$ & -2024.81 & $-8.70909 \times 10^{-9}$ & 99.6226 \\
\hline
\end{tabular}

\section{E Expansion of the PaVe functions around $m_{j}^{2}=0$}

The scalar PaVe functions involved in Eq. (22) are defined as follows

$$
\begin{aligned}
& B_{0}\left(p^{2}, m_{j}^{2}, m_{W}^{2}\right)=\left(i \pi^{2}\right)^{-1} \\
& \quad \times \int \frac{d^{n} k}{\left(k^{2}-m_{j}^{2}\right)\left[(k+p)^{2}-m_{W}^{2}\right]}, \\
& B_{0}\left(q^{2}, m_{j}^{2}, m_{j}^{2}\right)=\left(i \pi^{2}\right)^{-1} \int \frac{d^{n} k}{\left(k^{2}-m_{j}^{2}\right)\left[(k+q)^{2}-m_{j}^{2}\right]}, \\
& B_{0}\left(0, m_{j}^{2}, m_{W}^{2}\right)=\left(i \pi^{2}\right)^{-1} \int \frac{d^{n} k}{\left(k^{2}-m_{j}^{2}\right)\left(k^{2}-m_{W}^{2}\right)}, \\
& C_{0}\left(p^{2}, P^{2}, q^{2}, m_{j}^{2}, m_{W}^{2}, m_{j}^{2}\right)=\left(i \pi^{2}\right)^{-1} \\
& \quad \times \int \frac{d^{n} k}{\left(k^{2}-m_{W}^{2}\right)\left[(k+p)^{2}-m_{j}^{2}\right]\left[(k+P)^{2}-m_{j}^{2}\right]},
\end{aligned}
$$

where $p^{2}=m^{2}, P^{2}=M^{2}$ and $q^{2}=(P-p)^{2}=m^{2}+$ $M^{2}-2 P \cdot p$.

If we make an expansion around $m_{j}^{2}=0$, for the Eqs. (111, $112,113,114)$ following the same strategy that Cheng and Li for the $\mu \rightarrow e \gamma$ decay [13], we have that

$$
\begin{aligned}
& B_{0}\left(p^{2}, m_{j}^{2}, m_{W}^{2}\right) \approx B_{0}\left(p^{2}, 0, m_{W}^{2}\right) \\
& +m_{j}^{2} C_{0}\left(0, p^{2}, p^{2}, 0,0, m_{W}^{2}\right)+\vartheta\left(m_{j}^{4}\right), \\
& B_{0}\left(q^{2}, m_{j}^{2}, m_{j}^{2}\right) \approx B_{0}\left(q^{2}, 0,0\right) \\
& +2 m_{j}^{2} C_{0}\left(0, q^{2}, q^{2}, 0,0,0\right)+\vartheta\left(m_{j}^{4}\right), \\
& B_{0}\left(0, m_{j}^{2}, m_{W}^{2}\right) \approx B_{0}\left(0,0, m_{W}^{2}\right) \\
& +m_{j}^{2} \frac{A_{0}\left(m_{W}^{2}\right)}{m_{W}^{4}}+\vartheta\left(m_{j}^{4}\right), \\
& C_{0}\left(p^{2}, P^{2}, q^{2}, m_{j}^{2}, m_{W}^{2}, m_{j}^{2}\right) \approx C_{0}\left(p^{2}, P^{2}, q^{2}, 0, m_{W}^{2}, 0\right) \\
& +m_{j}^{2}\left[D_{0}\left(p^{2}, 0, q^{2}, P^{2}, p^{2}, q^{2}, m_{W}^{2}, 0,0,0\right)\right.
\end{aligned}
$$

$$
\begin{aligned}
& \left.+D_{0}\left(p^{2}, q^{2}, 0, P^{2}, P^{2}, q^{2}, m_{W}^{2}, 0,0,0\right)\right] \\
& +\vartheta\left(m_{j}^{4}\right) .
\end{aligned}
$$

Now, with the help of the Package-X program, we can obtain analytical expressions for the next functions

$$
\begin{aligned}
B_{0}\left(p^{2}, 0, m_{W}^{2}\right)= & \Delta+\frac{\left(p^{2}-m_{W}^{2}\right)}{p^{2}} \log \left(\frac{m_{W}^{2}}{m_{W}^{2}-p^{2}}\right) \\
& +\log \left(\frac{\mu^{2}}{m_{W}^{2}}\right)+2, \\
B_{0}\left(q^{2}, 0,0\right)= & \Delta+\log \left(-\frac{\mu^{2}}{q^{2}}\right)+2, \\
B_{0}\left(0,0, m_{W}^{2}\right)= & \Delta+\log \left(\frac{\mu^{2}}{m_{W}^{2}}\right)+1, \\
C_{0}\left(0, q^{2}, q^{2}, 0,0,0\right)= & -\frac{\Delta_{I}+\log \left(-\frac{\mu^{2}}{q^{2}}\right)}{q^{2}},
\end{aligned}
$$

with $\Delta_{I} \sim \Delta$ but associated with an infrared divergence.

$$
\begin{aligned}
C_{0}\left(0, p^{2}, p^{2}, 0,0, m_{W}^{2}\right)= & \frac{\Delta_{I}+\log \left(\frac{\mu^{2}}{m_{W}^{2}}\right)}{m_{W}^{2}-p^{2}} \\
& +\frac{\left(m_{W}^{2}+p^{2}\right)}{p^{2}\left(m_{W}^{2}-p^{2}\right)} \log \left(\frac{m_{W}^{2}}{m_{W}^{2}-p^{2}}\right),
\end{aligned}
$$

$$
\begin{aligned}
& D_{0}\left(p^{2}, 0, q^{2}, P^{2}, p^{2}, q^{2}, m_{W}^{2}, 0,0,0\right) \\
& =\frac{1}{q^{2}}\left[\frac{\Delta_{I}+\log \left(\frac{\mu^{2}}{m_{W}^{2}}\right)}{m_{W}^{2}-p^{2}}\right. \\
& +\frac{\left(m_{W}^{2}-P^{2}\right) \log \left(-\frac{m_{W}^{2}}{q^{2}}\right)}{-m_{W}^{2}\left(p^{2}+P^{2}-q^{2}\right)+m_{W}^{4}+p^{2} P^{2}} \\
& -\frac{\left(m_{W}^{2}-P^{2}\right) \log \left(\frac{m_{W}^{2}}{m_{W}^{2}-P^{2}}\right)}{-m_{W}^{2}\left(p^{2}+P^{2}-q^{2}\right)+m_{W}^{4}+p^{2} P^{2}} \\
& \left.+\frac{\log \left(\frac{m_{W}^{2}}{m_{W}^{2}-p^{2}}\right)\left(-m_{W}^{2}\left(p^{2}+P^{2}-2 q^{2}\right)+m_{W}^{4}+p^{2} P^{2}\right)}{\left(m_{W}^{2}-p^{2}\right)\left(-m_{W}^{2}\left(p^{2}+P^{2}-q^{2}\right)+m_{W}^{4}+p^{2} P^{2}\right)}\right],
\end{aligned}
$$


Table 9 Comparison for the $R_{a}$ factor using numerical fits vs Eq. (26)

\begin{tabular}{lll}
\hline Vertex & $R_{a}$ (Numerical Fits) & $R_{a}$ (Eq. 26) \\
\hline$Z \tau \mu\left(q_{\min }^{2}=4 m_{\mu}^{2}\right.$ for $\left.\tau^{-} \rightarrow \mu^{-} \mu^{-} \mu^{+}\right)$ & $11.5451+\mathrm{i} 3.4098$ & $11.3949+\mathrm{i} 3.14159$ \\
$Z \tau \mu\left(q_{\min }^{2}=4 m_{e}^{2}\right.$ for $\left.\tau^{-} \rightarrow \mu^{-} e^{-} e^{+}\right)$ & $22.2936+\mathrm{i} 3.40516$ & $22.0456+\mathrm{i} 3.14159$ \\
$Z \tau e\left(q_{\min }^{2}=4 m_{\mu}^{2}\right.$ for $\left.\tau^{-} \rightarrow e^{-} \mu^{-} \mu^{+}\right)$ & $11.5451+\mathrm{i} 3.4098$ & $11.3976+\mathrm{i} 3.14159$ \\
$Z \tau e\left(q_{\min }^{2}=4 m_{e}^{2}\right.$ for $\left.\tau^{-} \rightarrow e^{-} e^{-} e^{+}\right)$ & $22.2262+\mathrm{i} 3.40516$ & $22.0483+\mathrm{i} 3.14159$ \\
$Z \mu e\left(q_{\min }^{2}=4 m_{e}^{2}\right.$ for $\left.\mu^{-} \rightarrow e^{-} e^{-} e^{+}\right)$ & $31.6578+\mathrm{i} 1.15008$ & $22.7478+\mathrm{i} 3.14159$ \\
\hline
\end{tabular}

$D_{0}\left(p^{2}, q^{2}, 0, P^{2}, P^{2}, q^{2}, m_{W}^{2}, 0,0,0\right)$

$$
\begin{aligned}
= & \frac{1}{q^{2}}\left[\frac{\Delta_{I}+\log \left(\frac{\mu^{2}}{m_{W}^{2}}\right)}{m_{W}^{2}-P^{2}}\right. \\
& +\frac{\left(m_{W}^{2}-p^{2}\right) \log \left(-\frac{m_{W}^{2}}{q^{2}}\right)}{-m_{W}^{2}\left(p^{2}+P^{2}-q^{2}\right)+m_{W}^{4}+p^{2} P^{2}} \\
- & \frac{\left(m_{W}^{2}-p^{2}\right) \log \left(\frac{m_{W}^{2}}{m_{W}^{2}-p^{2}}\right)}{-m_{W}^{2}\left(p^{2}+P^{2}-q^{2}\right)+m_{W}^{4}+p^{2} P^{2}} \\
& \left.+\frac{\log \left(\frac{m_{W}^{2}}{m_{W}^{2}-P^{2}}\right)\left(-m_{W}^{2}\left(p^{2}+P^{2}-2 q^{2}\right)+m_{W}^{4}+p^{2} P^{2}\right)}{\left(m_{W}^{2}-P^{2}\right)\left(-m_{W}^{2}\left(p^{2}+P^{2}-q^{2}\right)+m_{W}^{4}+p^{2} P^{2}\right)}\right] .
\end{aligned}
$$

Replacing Eqs. $(115,116,117,118)$ and subsequently Eqs. $(121,119,120,123,122,123$ and 125) into (22) we obtain Eq. (24), with the $f_{Q}$ and $f_{R}$ factors given as follows

$$
\begin{aligned}
f_{Q_{a_{1}}}= & -q^{2}\left(-m^{2}-m M+m_{W}^{2}-M^{2}+q^{2}\right) \\
& \times\left(-m^{2}+m M+m_{W}^{2}-M^{2}+q^{2}\right), \\
f_{Q_{a_{2}}}= & \frac{1}{2 m^{2}}\left(\left(-\left(m^{2}-m_{W}^{2}\right)\left(m^{4}-m_{W}^{2}\left(m^{2}-M^{2}+q^{2}\right)\right.\right.\right. \\
& \left.\left.+m^{2}\left(M^{2}+q^{2}\right)-2\left(M^{2}-q^{2}\right)^{2}\right)\right), \\
f_{Q_{a_{3}}=} & \frac{1}{2 M^{2}}\left(-\left(M^{2}-m_{W}^{2}\right)\left(-2 m^{4}\right.\right. \\
& +m_{W}^{2}\left(m^{2}-M^{2}-q^{2}\right) \\
& \left.\left.+m^{2}\left(M^{2}+4 q^{2}\right)+M^{4}+M^{2} q^{2}-2 q^{4}\right)\right), \\
f_{Q_{a_{4}}=} & \frac{1}{2}\left(q^{2}\left(3\left(m^{2}+M^{2}-q^{2}\right)-2 m_{W}^{2}\right)\right), \\
f_{Q_{a_{5}}=} & \frac{1}{2}\left(\lambda\left(m^{2}, M^{2}, q^{2}\right) \log \left(\frac{\mu^{2}}{m_{W}^{2}}\right)\right. \\
& \left.+i \pi q^{2}\left(3\left(m^{2}+M^{2}-q^{2}\right)-2 m_{W}^{2}\right)\right) . \\
f_{R_{a_{1}}}= & 2 q^{2}\left(-m^{2}+m_{W}^{2}-M^{2}+q^{2}\right), \\
f_{R_{a_{2}}=}= & \frac{1}{m^{2} \alpha}\left(-m^{8}+2 m^{6}\left(m_{W}^{2}+q^{2}\right)\right. \\
& +m^{4}\left(-2 m_{W}^{4}+M^{4}-q^{4}\right) \\
& +m^{2} m_{W}^{2}\left(-2 q^{2} m_{W}^{2}+m_{W}^{4}-M^{4}+4 M^{2} q^{2}-3 q^{4}\right) \\
& \left.\left.-m^{2}\left(M^{2}-q^{2}\right)\left(m_{W}^{2}-M^{2}+q^{2}\right)\right)^{2}\right), \\
&
\end{aligned}
$$

$$
\begin{aligned}
f_{R_{a_{3}}}= & \frac{1}{M^{2} \alpha}\left(-M^{4}\left(\left(M^{2}-q^{2}\right)^{2}-m^{4}\right)\right. \\
& +m_{W}^{6}\left(-m^{2}+M^{2}+q^{2}\right) \\
& +2 m_{W}^{4}\left(m^{4}-2 m^{2} q^{2}-M^{4}-M^{2} q^{2}+q^{4}\right) \\
& +m_{W}^{2}\left(-m^{6}-m^{4}\left(M^{2}-3 q^{2}\right)\right. \\
& \left.\left.+m^{2}\left(4 M^{2} q^{2}-3 q^{4}\right)+2 M^{6}-3 M^{2} q^{4}+q^{6}\right)\right), \\
f_{R_{a_{4}}}= & \frac{1}{\alpha}\left(m^{6}-m^{4}\left(m_{W}^{2}+M^{2}+2 q^{2}\right)\right. \\
& +m^{2}\left(2 M^{2} m_{W}^{2}+q^{2}\left(q^{2}-m_{W}^{2}\right)-M^{4}\right) \\
& -M^{4}\left(m_{W}^{2}+2 q^{2}\right) \\
& +M^{2} q^{2}\left(q^{2}-m_{W}^{2}\right) \\
& \left.+2 q^{2} m_{W}^{2}\left(m_{W}^{2}+q^{2}\right)+M^{6}\right), \\
= & \frac{1}{\alpha}\left(i \pi \left(m^{6}-m^{4}\left(M^{2}+2 q^{2}\right)\right.\right. \\
& +m^{2}\left(q^{4}-M^{4}\right)-m_{W}^{2}\left(m^{4}+m^{2}\left(q^{2}-2 M^{2}\right)\right. \\
& \left.+M^{4}+M^{2} q^{2}-2 q^{4}\right) \\
& \left.\left.+2 q^{2} m_{W}^{4}+\left(M^{3}-M q^{2}\right)^{2}\right)\right),
\end{aligned}
$$

and $\alpha=m^{2}\left(M^{2}-m_{W}^{2}\right)+m_{W}^{2}\left(m_{W}^{2}-M^{2}+q^{2}\right)$.

Something remarkable at this point is:

The factor $Q_{a}$ has an ultraviolet divergence $\Delta$, as it can be seen in Eq. (25), but this divergence is independent of the neutrino mass. Then this divergence will vanish when we sum over the three families (GIM-mechanism), as it was mentioned previously.

Although there are infrared divergences $\Delta_{I}$ on the Eqs. (122, 123, 123, 125), the factor $R_{a}$ is free of them. Further, there is no dependence on the renormalization scale, and these results are in agreement with our numerical fits. Taking into account the imaginary part of the $C_{0}\left(m^{2}, M^{2}, q^{2}, 0, m_{W}^{2}, 0\right)$ function, it is possible to derive analytically that the imaginary parts appearing in the last column of Table 9 are exactly $\pi$.

\section{References}

1. S.L. Glashow, Nucl. Phys. 22, 579 (1961)

2. S. Weinberg, Phys. Rev. Lett. 19, 1264 (1967)

3. A. Salam, Conf. Proc. C 680519, 367 (1968) 
4. Y. Fukuda et al. [Super-Kamiokande Collaboration], Phys. Rev. Lett. 81, 1562 (1998)

5. Q.R. Ahmad et al. [SNO Collaboration], Phys. Rev. Lett. 87, 071301 (2001)

6. Q.R. Ahmad et al. [SNO Collaboration], Phys. Rev. Lett. 89, 011301 (2002)

7. N. Cabibbo, Phys. Rev. Lett. 10, 531 (1963)

8. M. Kobayashi, T. Maskawa, Prog. Theor. Phys. 49, 652 (1973)

9. B. Pontecorvo, Sov. Phys. JETP 10, 1236 (1960)

10. B. Pontecorvo, Zh Eksp, Teor. Fiz. 37, 1751 (1959)

11. Z. Maki, M. Nakagawa, S. Sakata, Prog. Theor. Phys. 28, 870 (1962)

12. S.L. Glashow, J. Iliopoulos, L. Maiani, Phys. Rev. D 2, 1285 (1970)

13. T.P. Cheng, L.F. Li, Gauge Theory Of Elementary Particle Physics (Oxford Science Publications, Oxford, 1984), p. 536

14. L. Calibbi, G. Signorelli, Riv. Nuovo Cim. 41, 1 (2018)

15. S.T. Petcov, Sov. J. Nucl. Phys. 25, 340 (1977) [Yad. Fiz. 25, 641 (1977); Erratum: Sov. J. Nucl. Phys. 25, 698 (1977); Erratum: Yad. Fiz. 25, 1336 (1977)]

16. B.W. Lee, R.E. Shrock, Phys. Rev. D 16, 1444 (1977)

17. X.Y. Pham, Eur. Phys. J. C 8, 513 (1999)

18. C. Patrignani et al. [Particle Data Group], Chin. Phys. C 40, 100001 (2016)

19. I. Esteban, M.C. González-Garcia, M. Maltoni, I. Martínez-Soler, T. Schwetz, JHEP 1701, 087 (2017)

20. F. Capozzi, E. Di Valentino, E. Lisi, A. Marrone, A. Melchiorri, A. Palazzo, Phys. Rev. D 95, 096014 (2017)
21. P.F. de Salas, D.V. Forero, C.A. Ternes, M. Tortola, J.W.F. Valle, Phys. Lett. B 782, 633 (2018)

22. Sw. Banerjee et al. [HFLAV-Tau group]. http://www.slac.stanford. edu/xorg/hflav/tau/spring-2017/tau-report-web.pdf. Accessed 25 Sept 2018

23. A.J. Buras. Weak hamiltonian, $\mathrm{CP}$-violation and rare decays, in Probing the Standard Model of particle interactions. In: Proceedings, summer school in theoretical physics, NATO advanced study institute, 68th session (Les Houches, France, 1997)

24. Belle II collaboration, E. Kou et al., The Belle II Physics Book, BELLE2-PUB-PH-2018-001. arXiv:1808.10567

25. T. Kinoshita, J. Math. Phys. 3, 650 (1962)

26. T.D. Lee, M. Nauenberg, Phys. Rev. 133, B1549 (1964)

27. G. Passarino, M.J.G. Veltman, Nucl. Phys. B 160, 151 (1979)

28. G. 't Hooft, M.J.G. Veltman, Nucl. Phys. B 153, 365 (1979)

29. R. Mertig, M. Bohm, A. Denner, Comput. Phys. Commun. 64, 345 (1991)

30. G.J. van Oldenborgh, J.A.M. Vermaseren, Z. Phys. C 46, 425 (1990)

31. T. Hahn, M. Pérez-Victoria, Comput. Phys. Commun. 118, 153 (1999)

32. Wolfram Research, Inc. Mathematica, Version 11.3 (Champaign, IL, 2018)

33. J.I. Illana, T. Riemann, Phys. Rev. D 63, 053004 (2001)

34. E. Arganda, A.M. Curiel, M.J. Herrero, D. Temes, Phys. Rev. D 71, 035011 (2005)

35. H.H. Patel, Comput. Phys. Commun. 197, 276 (2015) 